\title{
Acoustic Emission Monitoring of HFIR Vessel During Hydrostatic Testing
}

\section{Final Report}

\author{
M. A. Friesel
}

J. F. Dawson

August 1992

Prepared for the U.S. Department of Energy under Contract DE-AC06-76RLO 1830

Pacific Northwest L aboratory

Operated for the U.S. Department of Energy

by Battelle Miemorial Institute 


\title{
DISCLAIMER
}

This report was prepared as an account of work sporisored by an agency of the United States Government. Neither the United States Government nor any agency thereof, nor Battelle Memorial Institute, nor any of their employees, makes any warranty, expressed or implied, or assumes any legal liability or responsibility for the accuracy, completeness, or usefulness of any information, apparatus, product, or process disclosed, or represents that its use would not infringe privately owned rights. Reference herein to any specific commercial product, process, or service by trade name, trademark, manufacturer, or othenvise does not necessarily constitute or imply its endorsement, recommendation, or favoring by the United States Government or any agency thereof, or Battelle Memorial Institute. The views and opinions of authors expressed herein do not necessarily state or reflect those of the United States Government or any agency thereof.

\author{
PACIFIC NORTHWEST LABORATORY \\ operated by \\ BATTELLE MEMORIAL INSTITUTE \\ for the \\ UNITED STATES DEPARTMENT OF ENERCY \\ under Contract DE-ACO6.76RLO 1830
}

Printed in the United States of America

Avallable to DOE and DOE contractors from the

Office of Scientific and Technical Information, P.O. Box 62, Oak Ridge, TN 37831;

prices available from (615) 576.8401. FTS 626.8401.

Avaitable to the public from the National Technical Information Service,

U.S. Department of Commerce, 5285 Port Royal Rd., Springfteld, VA 22161. 
Final Report

M. A. Friesel

J. F. Dawson

August 1992

Prepared for

the U.S. Department of Energy

under Contract DE-AC06-76RLO 1.830

Pacific Northwest Laboratory

Richland, Washington 99352 


\begin{abstract}
This report discusses the results and conclusions reached from applying acoustic emission monitoring to surveillance of the High Flux Isotope Reactor vessel during pressure testing. The objective of the monitoring was to detect crack growth and/or fluid leakage should it occur during the pressure test. The report addresses the approach, acoustic emission instrumentation, installation, calibration, and test results.
\end{abstract}




\section{Contents}

Abstract $\ldots \ldots \ldots \ldots \ldots \ldots \ldots \ldots \ldots \ldots \ldots \ldots \ldots \ldots \ldots \ldots \ldots \ldots \ldots$

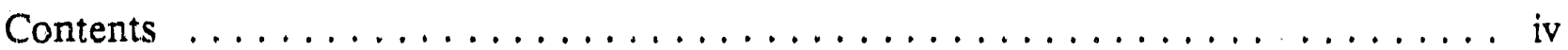

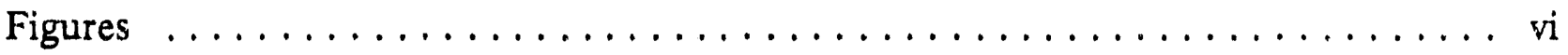

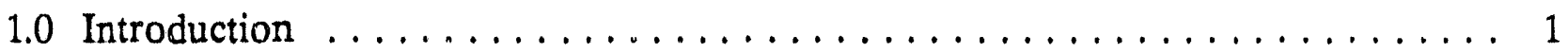

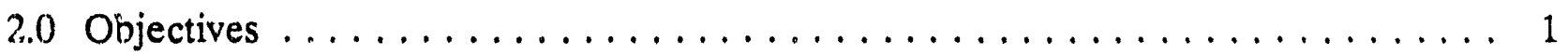

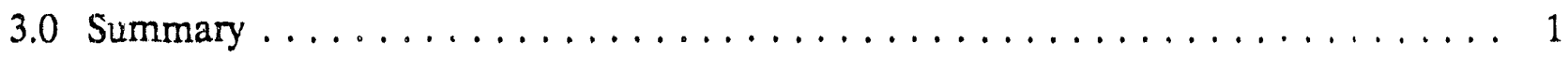

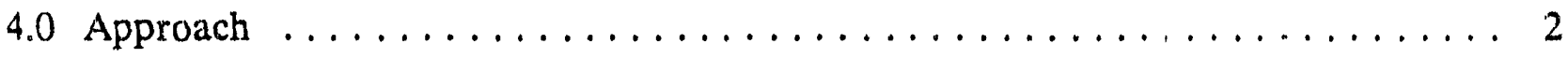

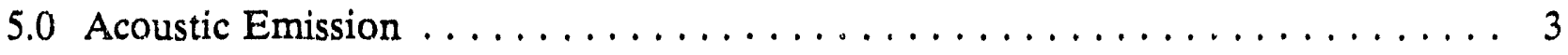

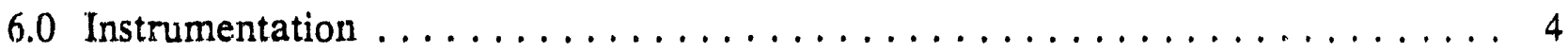

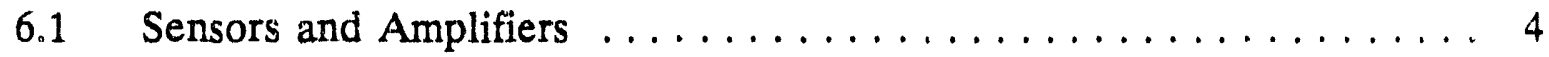

6.1 .1 Physical Description . . . . . . . . . . . . . . . . . 4

6.2 Data Acquisition and Analysis Instrumentation $\ldots \ldots \ldots \ldots \ldots$

7.0 Sensor Installation $\ldots \ldots \ldots \ldots \ldots \ldots \ldots \ldots \ldots \ldots$

8.0 ASTM Standards $\ldots \ldots \ldots \ldots \ldots \ldots \ldots \ldots \ldots \ldots \ldots \ldots$

9.0 Test Preparation $\ldots \ldots \ldots \ldots \ldots \ldots \ldots \ldots \ldots \ldots \ldots \ldots \ldots \ldots$

9.1 Pulser Tests and Sensor Response Evaluation: Pre- and PostHydro test ........................... 11

9.2 Test Procedure . . . . . . . . . . . . . . . . . . . 13

9.3 Instrument Settings $\ldots \ldots \ldots \ldots \ldots \ldots \ldots \ldots \ldots \ldots \ldots \ldots$

10.0 Test Results . . . . . . . . . . . . . . . . . . . . . . . 15

10.1 General ................................. 15

10.2 Description of Acoustic Emissions .................. 15

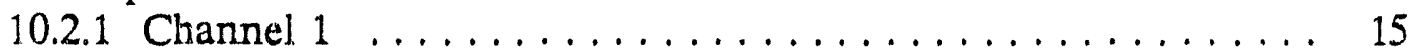

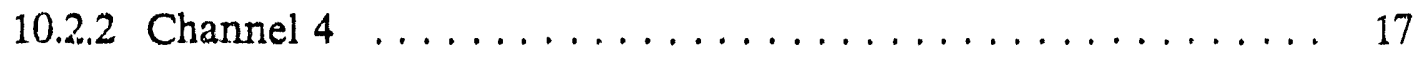

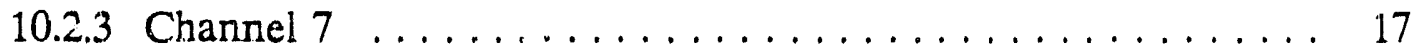

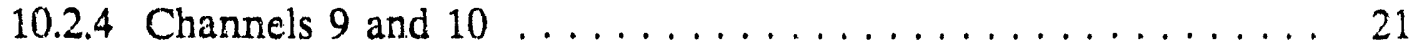


11.0 Evaluation of Test Results $\ldots \ldots \ldots \ldots \ldots \ldots \ldots \ldots \ldots \ldots \ldots \ldots \ldots \ldots \ldots \ldots, 22$

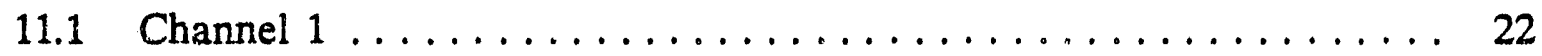

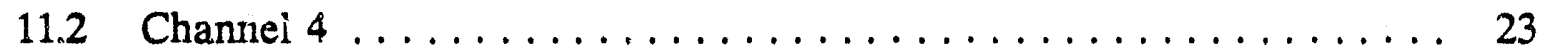

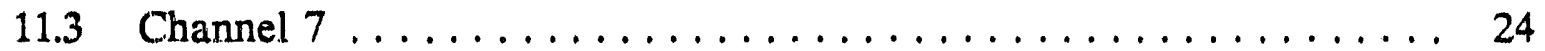

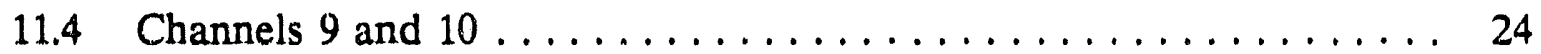

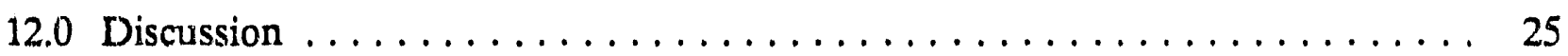

12.1 Noise and Instrumentation Effects $\ldots \ldots \ldots \ldots \ldots \ldots \ldots \ldots, 26$

12.2 Instrumentation Effects on Acoustic Emissions . . . . . . . . . 27

12.3 Peculiarities of the Waveguide Sensor Response . . . . . . . . . . 29

12.4 Calibration and Real Acoustic Emission ................. 30

12.5 Interpretation and Applicability of ASTM Standard E 569 and E

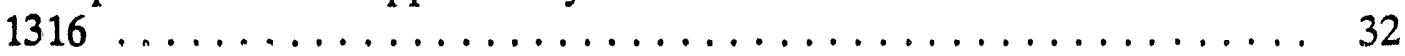

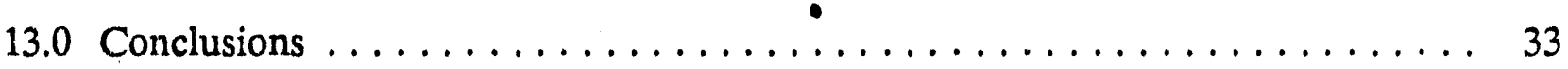

14.0 Acknowledgements $\ldots \ldots \ldots \ldots \ldots \ldots \ldots \ldots \ldots \ldots \ldots \ldots \ldots \ldots \ldots \ldots \ldots, 33$

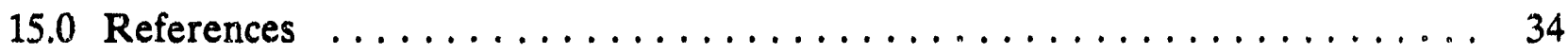

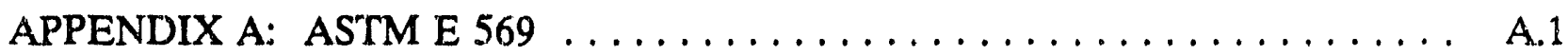

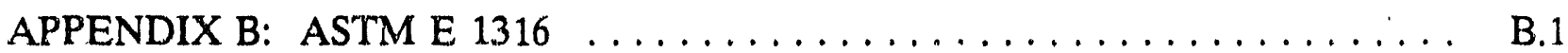




\section{Figures}

1. Schematic of a Typical Waveguide Sensor Employed at ORNL .........

2. Representative Waveguide Sensor Response to Helium Gas Jet Excitation on a Calibration Block ............................ 6

3. Pulser Mounted on the Extension Handle - Sketch . . . . . . . . . . . . . 7

4. Pressure Vessel Sidewall Roll-Out with Sensor and Pulser Locations

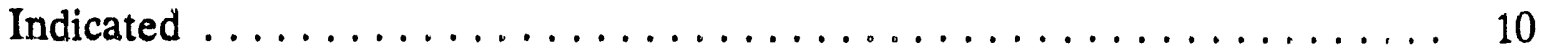

5. Pressure Head Diagram Showing Stud Numbering and Beam Tube

6. Cumulative Acoustic Emission Events and Applied Pressure as a Function of Time f.or All Active Channels . . . . . . . . . . . . . . . . . . . . . 16

7. Sample of Raw Data Obtained During High Channel 7 Event Rate . . . . . 18

8. Illustration of the Periodicity in Channel 7 Events $\ldots \ldots \ldots \ldots \ldots \ldots$

9. Channel 7 Data with Periodic and Internal Reflection Events Removed Overlayed on the Unfiltered Data shown in Figure $6 \ldots \ldots \ldots \ldots$

\section{Tables}

1. Comparison of Lead Break and Pulser AE Sources $\ldots \ldots \ldots \ldots \ldots \ldots, 7$

2. Minimum Detectable Input Signal Amplitudes and Range of Displayed

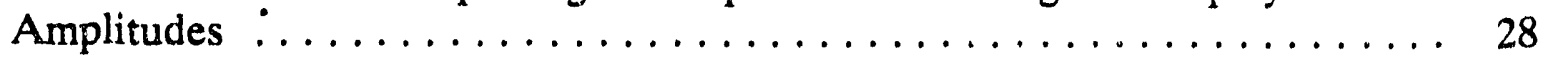

3. Estimated Arrival Times for Internally Reflected Wavemodes in a 17'8" Waveguide Sensor ................................. 29

4. Example Relative Arrival Times of Low-Amplitude Channel 7 Events Subsequent to High-Amplitude Events 


\subsection{Introduction}

The High Flux Isotope Reactor (HFIR) located at Oak Ridge National Laboratory contains four "beam tubes" which penetrate the reactor vessel wall and continue to the reactor core. Inherently, these tubes provide a path whereby neutrons from the core can strike a small area of the reactor vessel wall around the beam tube nozzles with the resulting potential for embrittlement of the steel. Periodic hydrostatic overpressure tests have been implemented as a precaution to ensure the safety of the pressure vessel, and have been complemented by implementation of acoustic emission monitoring to detect active crack growth. Acoustic emission (AE) is also capable of detecting high- pressure fluid leakage through cracks and seals, which produces signals distinct from those of crack growth.

In 1987 the Pacific Northwest Laboratory (PNL) performed AE monitoring during hydrostatic pressurization of the HFIR vessel. The present report discusses the results of the second AE monitoring test performed by PNL at ORNL during hydrostatic testing of the HFIR pressure vessel in March 1992.

The expected behavior of crack-growth acoustic emissions, the chief source of interest, has been used as the primary reference behavior throughout this report. Any apparent correlation between this behavior and that of detected acoustic emissions should not be interpreted as other than an indication.

\subsection{Objectives}

The objective of monitoring the HFIR pressure vessel with AE during hydrostatic testing is to detect, identify, and locate any crack growth or leakage of pressurizing fluid occurring during the test.

\subsection{Summary}

The HFIR vessel was pressure tested while being monitored for acoustic emissions on March 5, 1992. Pressure was applied in a step-and-hold format reaching a maximum of $925 \pm 12.5$ psi as measured by the parametric input of the acoustic emission monitoring system. Significant acoustic emission indications were obtained from the following channels: 
- channel 1, monitoring the upper part of the vessel above HB-1

- channel 7, monitoring the upper part of HB-3

- channels 9 and 10, covering the upper and lower parts of HB-4 respectively

- a much lower, but still significant, number of events was obtained from channel 4 located on the upper part of HB-2.

Other channels produced few or no indications. Channel 10 data has a strong likelihood of being predominantly noise internal to the $\mathrm{AE}$ instrument.

The sources of detected acoustic emissions remaining after noise removal have not been identified. Although some behavior of the data is not inconsistent with both crack growth and leakage, it is equally likely that the observed emissions arise from innocuous sources. Therefore any proposed source of these emissions should be regarded as speculative.

While the sources of AE detected by active channels may be classified as active, no data can clearly be classified as intense, which is the lowest ASTM category for which supplemental inspection using other NDT methods is recommended (1991 Annual Book of ASTM Standards [1]). There is also some likelihood that emissions detected by channels 1 and 9 are due entirely or in part to leakage.

No source location using triangulation techniques was possible, since each signal was detected only by a single sensor. This condition invalidates a very powerful tool for source identification, but it is likely that acoustic emission sources must be subsurface and located within a few feet of the active channel, with the possible exception of channel 1.

\subsection{Approach}

The approach taken by PNL in general paralleled that used in the 1987 test as described in the final report for that work [2]. As the conditions of the test had not significantly changed, much of the test configuration, equipment, and procedures were also the same as used previously.

Acoustic emission waveguide sensors were attached to the HFIR pressure vessel at specified locations as described below, to ensure coverage of critical areas. Acoustic emission from all sensors was monitored continuously during the pressurization test of the vessel using a commercial AE monitoring instrument (SPARTAN $3000^{\circ}$ marketed by 
Physical Acoustics Corporation). Sensitivity of the entire AE system was verified by monitoring calibration signals from an acoustic pulser placed at different locations on the vessel wall. This verification was performed both before and after the pressurization test.

\subsection{Acoustic Emission}

Acoustic emissions are transient elastic displacements of a surface usually caused by a remote, localized source comparable to an earthquake except for scale. The emissions are typically comprised of a few positive- and negative-going spike-like displacements of varying amplitude, radiating symmetrically from the point of origin. Separation between the most energetic of these displacements in a given signal is up to a few tens of microseconds over typical monitoring distances in acoustic emission applications. These signal components are the dispersed and attenuated wavemodes appropriate to the geometry and material of the test specimen, to the loading conditions, to the source and sensor locations, and the source type and orientation. Modes are distinguished by the direction of material displacement relative to the direction of wave propagation, and have distinct propagation speeds. In thin materials, the fundamental symmetric and anti-symmetric wavemodes predorninate, while in thick materials the longitudinal and shear waves may be detected, or a single Rayleigh wave may be the only detectable mode at large source/sensor separations.

Piezoelectric transducers are commonly used to detect these waves. The transducer is mounted to a surface of the specimen being monitored and converts any detected displacements into a covariant voltage. After amplification, the voltage signal is processed and analyzed by appropriate instrumentation.

Detailed analysis of the signals may reveal a great deal of information about the source, but this approach is unsuitable for many practical applications at present. Standard acoustic emission analysis such as that employed in the 1987 test of the HFIR pressure vessel [2] and in the current test relies on identifying characteristics which generally describe the envelop of the sensor response to the detected signal, along with a limited amount of other information as described below. 


\subsection{Instrumentation}

\subsection{Sensors and Amplifiers}

Waveguide sensors were used throughout in order to protect electronic components from exposure to water.

\subsubsection{Physical Description}

Eight of the ten waveguide sensors were those developed and employed in the 1987 test. Two additional sensors were manufactured by PNL to the same specifications as the others, to replace dysfunctional sensors. The sensors were formed by bonding a piezoelectric crystal to one end of a 17 foot 8 inch by $1 / 8$ inch diarreter stainless steel rod. This end of the rod was enclosed to protect the crystal and an integral preamplifier. Figure 1 illustrates the components and construction of a typical sensor.

The sensor/pre-amplifier systems were designed to provide peak sensitivity between 300 $\mathrm{kHz}$ and $350 \mathrm{kHz}$, and show high roll-off below about $150 \mathrm{kHz}$ in order to facilitate low frequency noise rejection. The pre-amplifiers provided a nominal $20 \mathrm{~dB}$ of gain and were placed near the piezoelectric crystal to diminish noise. Combined with nominal 40 $\mathrm{dB}$ of additional broadband amplification, the total gain entering the $\mathrm{AE}$ monitoring system was $60 \mathrm{~dB}$. Attenuation in the waveguides was measured to be about $8 \mathrm{~dB}$.

\subsubsection{Acoustic Emission Sensor Characterization}

Acoustic emission sensor characterization was performed at PNL on newly constructed sensors, while sensors remaining from the 1987 test where evaluated at Oak Ridge by ORNL personnel using PNL procedures. These procedures are identical to those used during the 1987 program, and consist of comparison of background electronic noise level with the response obtained from excitation of the waveguide sensors. The waveguide sensors were excited using a helium gas jet from a 13 psig source forced through a \#18 hypodermic needle applied to the end of the waveguide with a 1/8-inch standoff of the needle tip. The results from this calibration test give a sensor frequency response piofile and a relative measure of sensor sensitivity to permit a comparison of sensor performance among a group of sensors.

A representative calibration result for frequencies from 0 to $1 \mathrm{MHz}$ is illustrated in Figure 2. Some greater variability was observed in the response characteristics of the sensors when compared to the 1987 calibration data. The peak response frequency determined from the latest calibration varied between about 250 and $450 \mathrm{kHz}$ while 


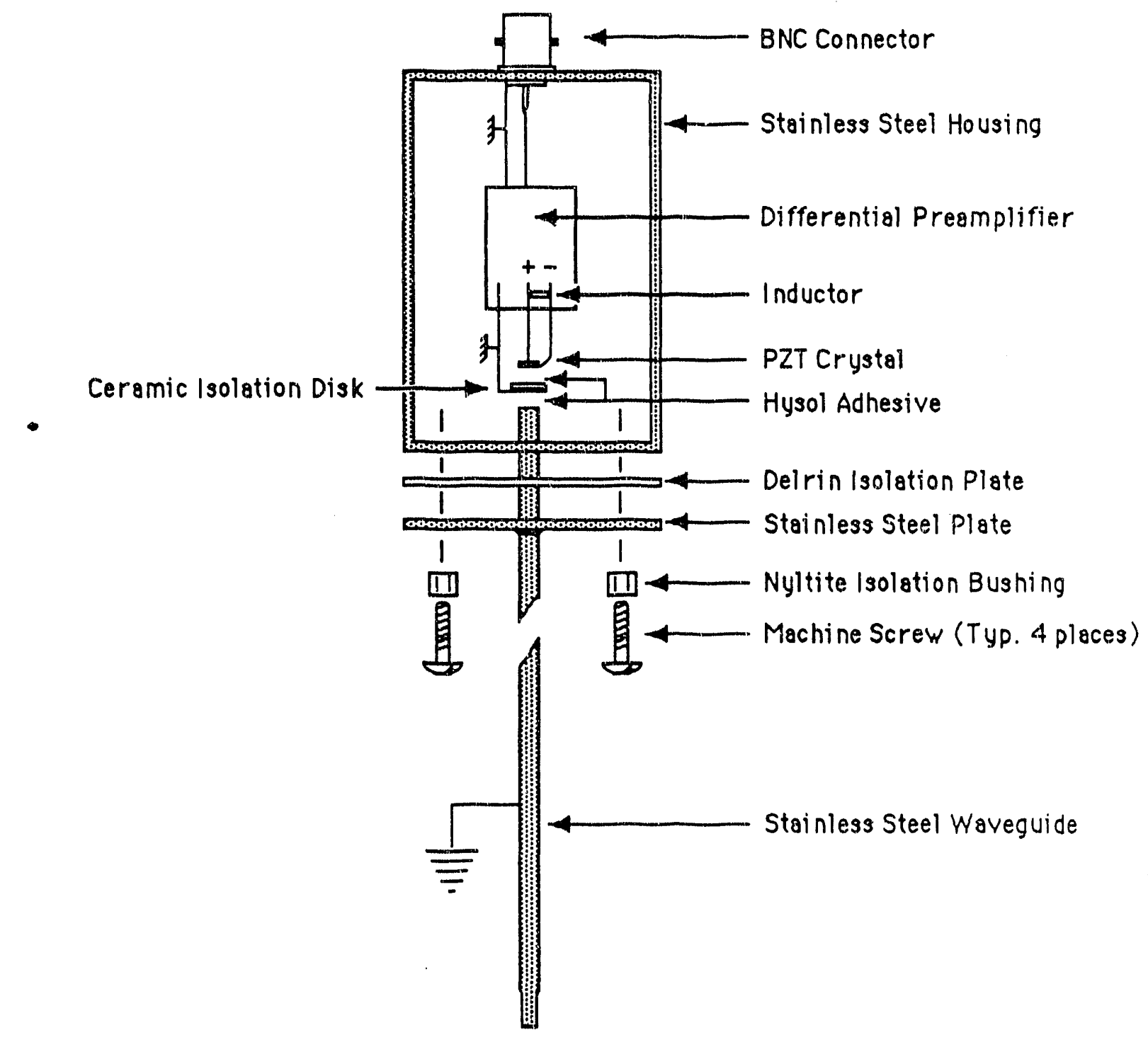

Figure 1. Schematic of a Typical Waveguide Sensor Employed at ORNL

background noise levels were slightly higher than previously measured, resulting in decreased signal-to-noise. Frequency roll-off is about $150 \mathrm{kHz}$.

Sensitivity was also examined with the sensors in situ both before and after the pressure test was run. An electronic pulser was mounted on the end of an extension handle constructed by ORNL personnel as shown in Figure 3, which permitted the pulser to be placed at various locations around the vessel wall. The pulser is a piezoelectric sensor which produces transient acoustic signals suitable for functional testing of $\mathrm{AE}$ instruments. During response tests which preceded and followed the pressure vessel test, the pulser was activated at each location and the response of the acoustic emission 


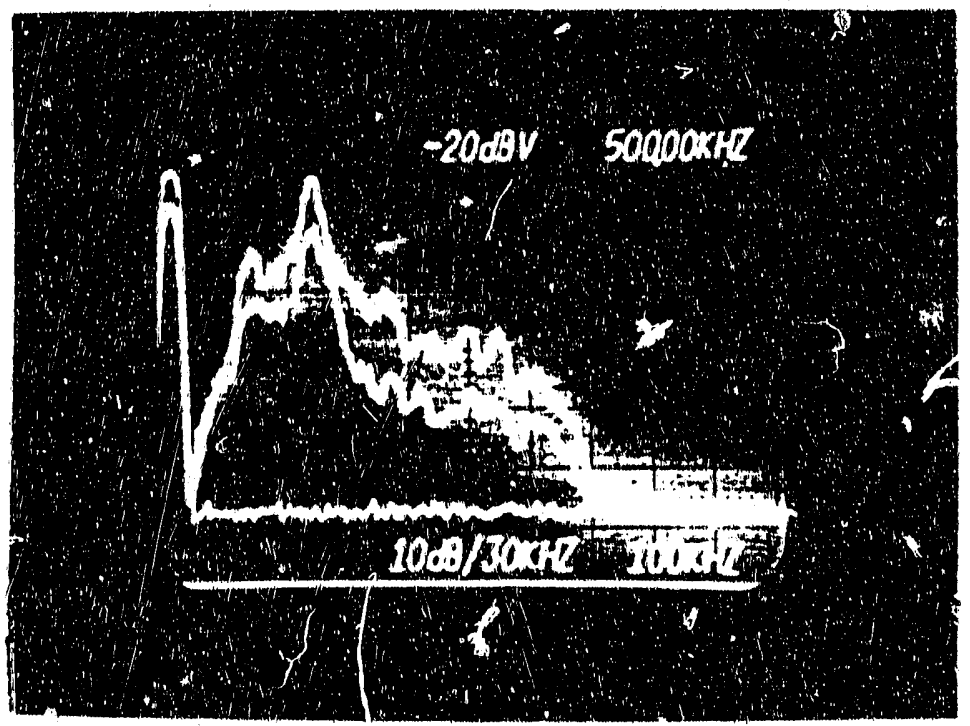

Figure 2. Representative Waveguide Sensor Response to Helium Gas Jet Excitation on a Calibration Block. Lower sace electronic background; middle trace - radial excitation near the waveguide tip; upper trace - excitation in the axial direction of the waveguide.

iystem to these signals examined and evaluated. Pulser and sensor positions and method oi placement are described below.

Coniparison between the pulser signal and the signal produced by breaking 0.7 pencil lead was also carried out at the request of an ORNL representative. The breaking of a pencil lead is a widely used and well- characterized transient acoustic emission calibration standard. This experiment was set-up by attaching a waveguide sensor to a $12 \times 12 \times 4$ inch carbon steel test block with $50 \mathrm{lbs}$. pressure applied to the waveguide to provide good contact with the surface of the block. The signal produced by breaking the pencil lead using a mechanical pencil at a four inch distance from the sensor was compared to the signal from a water-coupled pulser at the same location. The $0.7 \mathrm{~mm}$ lead was used instead of the 0.5 lead used in previous tests because the proper type of smaller lead was not available. The pulser was similar in design to that used for in situ sensor calibration, and driven by both $100 \mathrm{~V}$ and $300 \mathrm{~V}$ transient pulses for this experiment. The results showed the ratio of the detected pencil lead peak-to-peak amplitude to those of the $100 \mathrm{~V}$ and $300 \mathrm{~V}$ pulser signals to have the ratios $1.00 / 1.35$ and 1.00/3.62 respectively as shown in Table 1. 


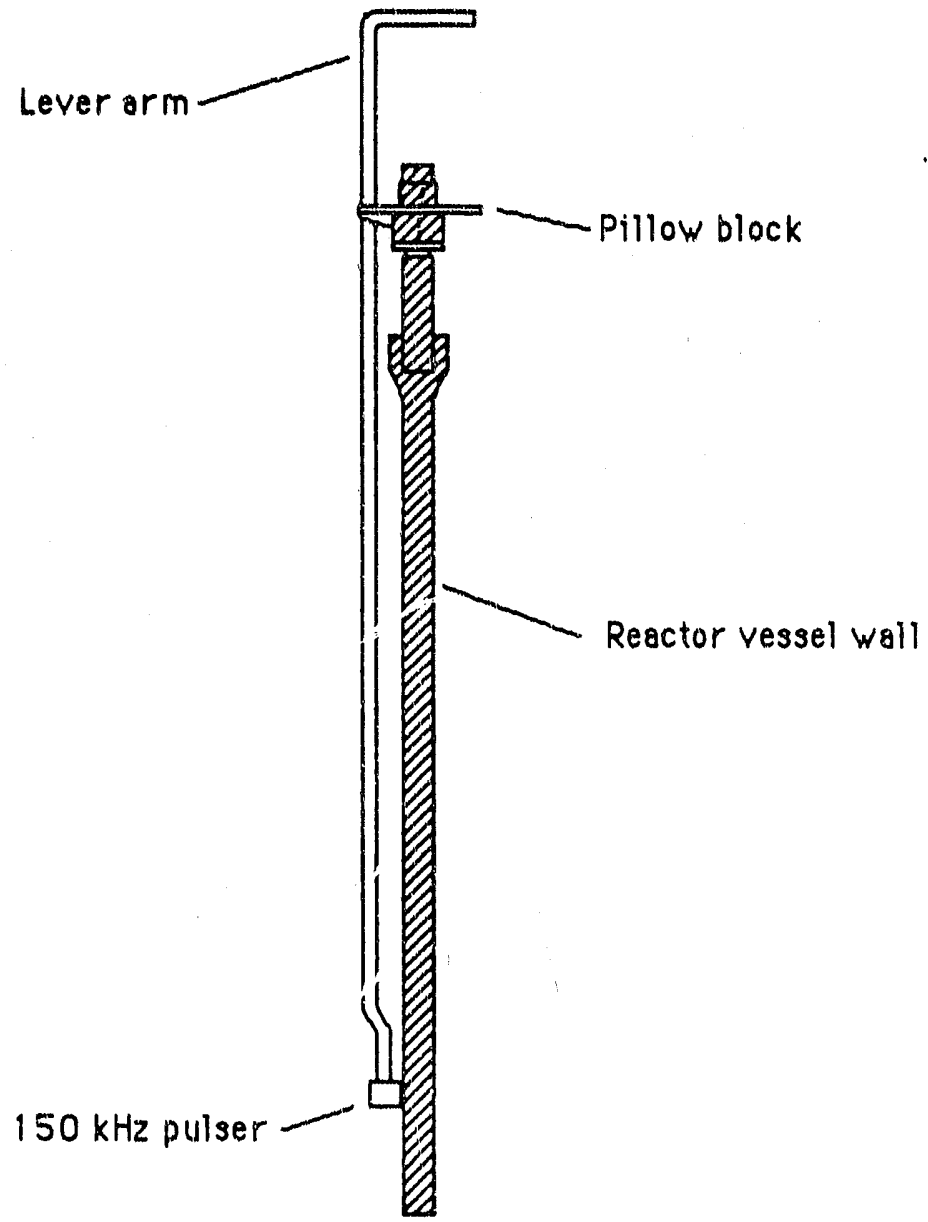

Figure 3. Pulser Mounted on the Extensicn Handle - Sketch

Table 1. Comparison of Lead Break and Pulser AE Sources

\begin{tabular}{|c|c|}
\hline Source & Volts $(\mathrm{p} / \mathrm{p})$ \\
\hline 0.7 -mm Lead & 2.18 \\
\hline 100 V Pulser & 2.95 \\
\hline 300 V Pulser & 7.90 \\
\hline
\end{tabular}


The injection losses in the test block are expected to be somewhat lower than on the vessel wall, due to the effects of the curved surface and weld-bead cladding on the vessel, and difficulties inherent in remote positioning on the quality of the pulser contact.

The amplitude of a pencil lead break signal changes as the amount of applied force necessary to break it. In contrast to the 1987 results, the pencil lead is the lowest of the three inputs despite the larger diameter of the lead.

It is significant that the acoustic signals pioduced by breaking a pencil lead may exceed those produced by microscopic crack growth by an order of magnitude or more. The pencil lead emissions detectable by a sensor mounted on the same surface of a thick component as the source are surface waves, and of these waves the non-dispersive Rayleigh wave is usually the most significant component.

\subsection{Data Acquisition and Analysis Instrumentation}

Acoustic emission sensors detect surface displacements and convert them to electrical signals. Commercial sensors typically have a narrow peak response frequency band and low damping, hence the response of the sensor to spike-like displacements tends to appear as a short rise-time signal with an exponentially decaying tail, oscillating near the sensor resonant frequency. The effect of a long, cylindrical waveguide is to convert a displacement spike into rod modes and to attenuate the signal. Signal energy loss is also aggravated by the waveguide/surface interface. Rod modes may be internally reflected and these reflections interpreted as distinct events. Effects of rod modes and internai reflections on acoustic emission analysis are discussed further in Section 12.

The sensor response is amplified and filtered. Initial filtering is caused by conversion of the surface modes to the rod modes of the waveguide sensor, but filtering primarily occurs because of the transducer sensor response function which preferentially amplifies certain frequencies and rejects those outside of the passband. Additional frequency filtering is usually performed electronically. Signals are detected when the amplified sensor output exceeds a pre-set voltage threshold, hence signals are also amplitude filtered prior to processing. The details of filtering and other analysis functions are device-dependent in practice.

The instrument used for data acquisition and analysis was a SPARTAN $3000^{\circ}$ obtained on lease from Physical Acoustics Corporation. The instrument calculates standard AE signal parameters and stores them on diskette, along with parametric input. Graphs of data trends can be displayed during acquisition. Pertinent characteristics derived and recorded by the instrument include the signal arrival time, the event sequence number which is simply the order of occurrence of the event relative to other events, amplitude, duration, rise time, energy, and ringdown counts. The system is also capable of calculating and displaying source location information. 
Signal amplitude is the peak amplitude of the signal measured from the threshold level. The amplitude is displayed in decibels with a range from zero to $100 \mathrm{~dB}$. Signals with amplitude exceeding $100 \mathrm{~dB}$ are clipped but retained, while those with amplitude below threshold are rejected. Duration as measured by the SPARTAN is the elapsed time from the time of the peak amplitude measurement until the end of the event. The signal rise time is length of time from the threshold crossing until the peak signal voltage (i.e. signal amplitude) is reached. Energy is typically determined as the product of the squared peak voltage and the signal duration, while counts are the number of times the oscillating signal crosses the threshold. Parametric inputs allow non-acoustic emission data to be sampled either periodically or simultaneously with the occurrence of detected events. Parametric inputs must be in the range of 0 to 5 volts. The SPARTAN parametric was used to measure a 0 to 5 volt pressure gauge signal, providing a resolution of $0.1 \mathrm{~V}$.

\subsection{Sensor Installation}

Locations of the AE sensors are shown in Figure 4. The sensor locations, mounting fixtures, and related hardware, were the same as or identical to those used in the 1.987 test. Sensors were located above and below the beam tube nozzles, and two sensors were located near the top of the vessel as illustrated in the figure. ORNL staff again performed the sensor installation.

\subsection{ASTM Standards}

Three ASTM standards applied to AE monitoring of the HFIR pressure vessel. The primary one is E569, "Standard Practice for Acoustic Emission Monitoring of Structures During Controlled Stimulation" [1]. E569 references E750, "Standard Practice for Measuring the Operating Characteristics of Acoustic Emission Instrumentation" [1]. Both of these standards were followed to the degree technically practical. Standard E1316 is a listing of definitions. Standards E569 and E1316 are included as Appendices to this report.

Due to difficulty in obtaining the monitoring instrument, it was shipped directly to Oak Ridge, arriving two days prior to installation. This did not allow time for a field engineer from Physical Acoustics Corporation to check-out and calibrate the instrument on-site, hence as much check-out as possible was performed by the operators. Calibration stickers indicated that the acoustic emission computer component of the instrument had been calibrated in January 1991, while the remaining component had been calibrated in February 1992. 


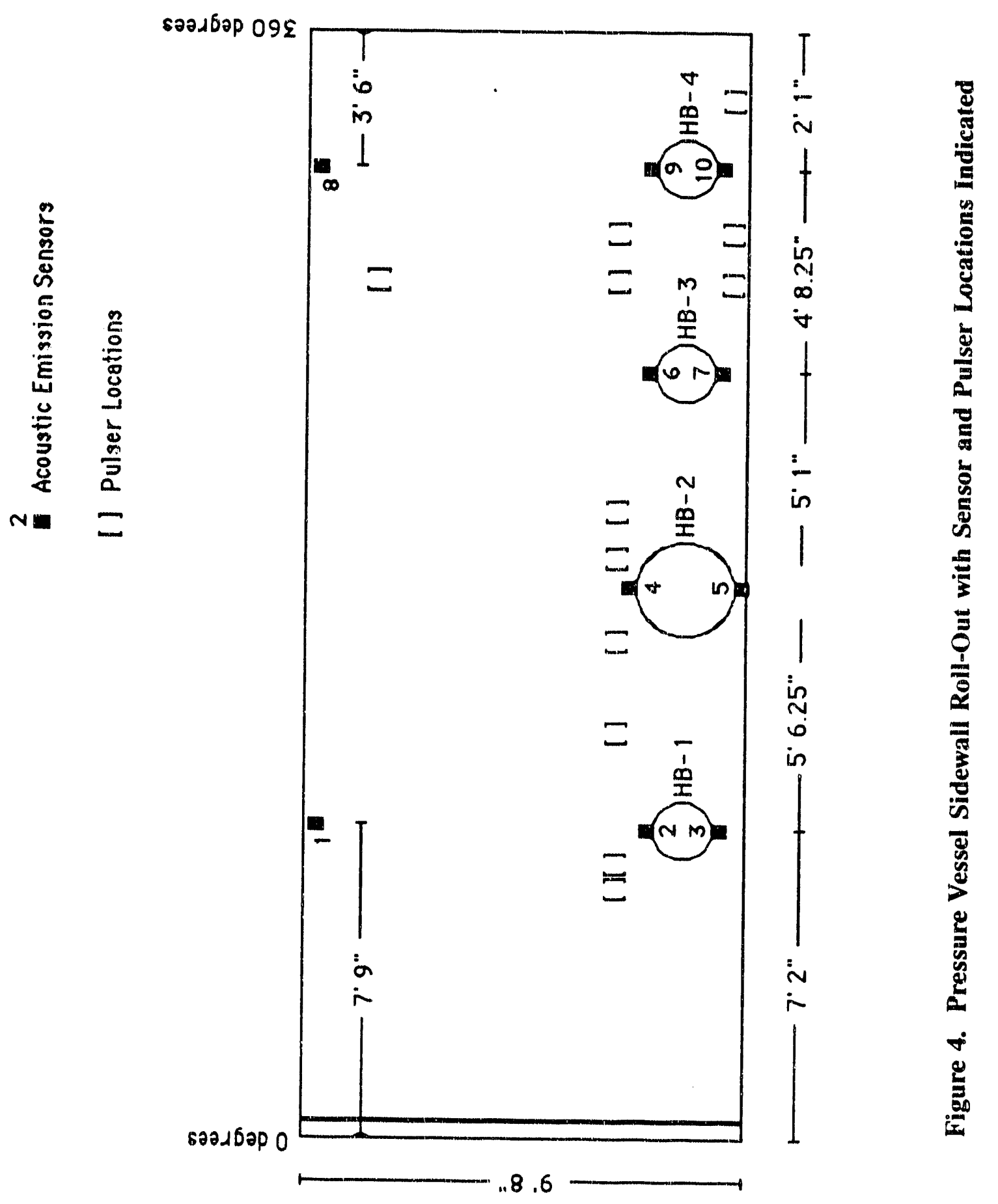


Notes regarding calibration presented in the 1987 test results are applicable to the current tests. As an alternate to the ASTM-specified calibration, an electronic pulser mounted on an extension handle was pressed against the side of the vessel at selected points to inject signals to test the sensitivity and source location capability of the $\mathrm{AE}$ system. The pulser signal strength was compared separately to the signal produced by breaking pencil leads. This is discussed further below.

\subsection{Test Preparation}

\subsection{Pulser Tests and Sensor Response Evaluation: Pre- and Post- Hydro-test}

A high water level in the well surrounding the main reactor vessel was maintained during the sensor response tests. The detection threshold for the pre-pressurization series of response evaluation tests was set at $65 \mathrm{~dB}$ for all eight shannels, while the post-pressurization evaluation test thresholds were held at $55 \mathrm{~dB}$ corresponding to test threshold levels. The pulser was placed in the vicinity of the locations indicated by brackets in Figure 4. The pulser had to be positioned by an operator standing on a walkway above the pressure vessel, therefore in order to assure reasonable accuracy in the pulser positions the pulser rod was marked to indicate vertical distance. Azimuthal position was determined by numbering the head bolts as illustrated in Figure 5 and noting the number which corresponded to the position of the pulser. After positioning, the pulser was activated and the response of the sensors recorded. Response to both 300 $\mathrm{V}$ and a less complete set of $100 \mathrm{~V}$ pulser excitations were examined.

During both pre- and post- test sensor response evaluations, of the ten sensors only channel 8 was insufficiently sensitive to respond to any input. Channel 5 was also found to be insensitive. Although at some positions channel 5 detected pulser events, there were no detected events with amplitude below $60 \mathrm{~dB}$ despite a threshold setting of 55 $\mathrm{dB}$. This observation has been ascribed to poor calibration of the SPARTAN for reasons discussed in detail below.

Data from each channel was examined to ensure proper functioning of the instrument, and to identify the probable propagation path of the detected signal. The time of occurrence of each event was recorded with one-tenth microsecond resolution. This time is provided by a clock marking the elapsed time from the start of the test, hence the difference in arrival time of a signal at a given pair of sensors can be found by subtracting these arrival times. Possible propagation paths were determined from the pulser location and the sensor locations, and the wave speeds calculated. For example, the delta-times between channels 6 and 7 were calculated when the puiser was located at lug 33 (see Figure 5) at mid-level (80 inches below the upper girth weld shown in 


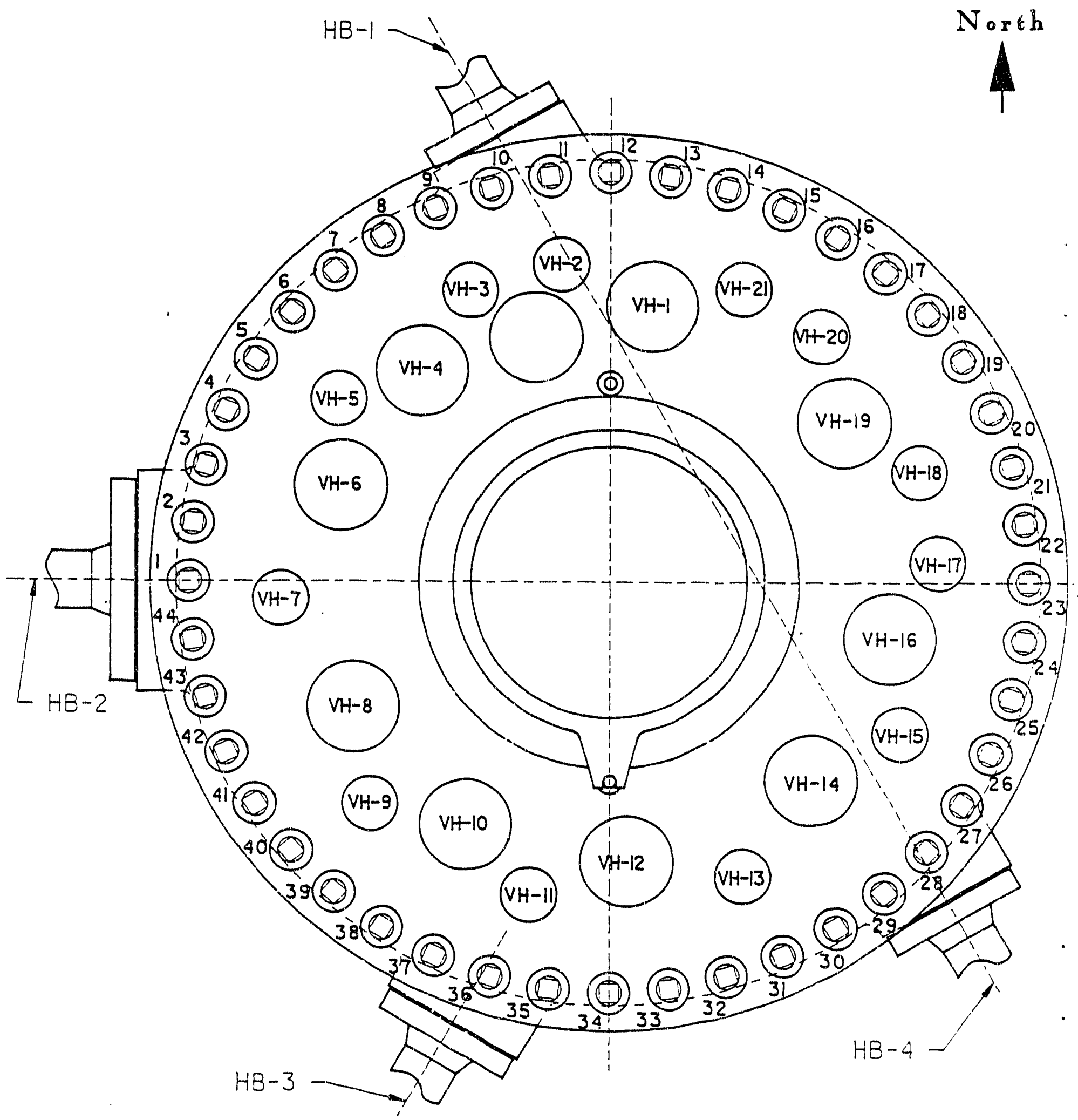

Figure 5. Pressure Head Diagram Showing Stud Numbering and Beam Tube Locations 
Figure 4). In this set, twelve delta-times in this set of data were found to exhibit reasonable and similar values for the known sensor separation, while other possible hit sequences produced delta-times which were much too large for any likely wavemode. The mean delta-time of the 12 signals was calculated to be 231.6 microseconds with a standard deviation of about 16 microseconds. Approximate distances for the most direct geometric paths along the vessel surface from the source to the two waveguide tips were then determined taking into account the nozzle curvature. Distances were also calculated for the direct water path to these sensors. The difference between source-sensor surface paths in this example was found to be about 21 inches, giving an approximate wave speed of 0.093 inches per microsecond. This value is in the range of surface wave speeds in steels. By the direct water path, however, sensor 6 cannot detect the signal first, contrary to the data, except where the waveguides are close together as they run up the attachment arm. In this latter case the delta-times should be no more than one or two microseconds It is probable therefore that the surface wave is detected by these sensors.

With the pulser at mid-level on lug 42, the delta-times between channels 6 and 7 fell into two groups. One group showed delta-times near 220 microseconds which, by again calculating the most direct geometric path along the vessel surface, produced wave speeds of about $0.04 \mathrm{in} / \mu \mathrm{sec}$. An error of only a few inches in the pulser or receiver placement is sufficient to raise this value to that of water. The second group, with delta-times of about 1 microsecond, produced wave speeds near $10 \mathrm{in} / \mu \mathrm{sec}$ using these same distances. This second wave speed is far too high, hence these signals either are noise or else the real or effective sensor separation is substantially less than the distance between the tips of the waveguides. The signals corresponding to these latter wave speeds are not distinguishable from other events on the basis of recorded signal parameters, therefore a reasonable explanation is that the signals were detected at a point where the sensor separation was only about $1 / 2$ inch or so. This condition occurs on the sensor mounting fixture and indicates a water path for signal propagation. Because of these results, it appears that a surface wave propagating through the vessel wall or an acoustic wave propagating through water may be detected, depending on the distance of the sensor from the source and possibly other geometric effects.

\subsection{Test Procedure}

The test plan specified that pressure would be increased in steps from a $100 \mathrm{psi}$ minimum to a maximum of 900 psi. Pressure would be increased in 100 psi increments up to $800 \mathrm{psi}$, followed by a 50 psi increment and two 25 psi increments to maximum pressure. Background acoustic emission would be measured at 100 psi. The water level in the pressure vessel well was reduced to below that of the lowest acoustic emission sensors prior to beginning the test. 
Pressure transitions during testing were accomplished in under two minutes. Background was measured for ten minutes while $\mathrm{AE}$ was monitored during pressure increases and for five minute periods at each static pressure. Interruptions in testing were necessary in order to remove water from the vessel well, when a remote camera was operated, and because of a pressure gauge which was improperly calibrated above 500 psi. Acoustic emission monitoring was disabled during these interruptions due to the increased noise levels.

The actual pressures achieved during the test, as determined from parametric input to the acoustic emission instrumentation, followed the above description to $400 \mathrm{psi}$. Instead of a $100 \mathrm{psi}$ increment to $500 \mathrm{psi}$, the subsequent measured pressure reached $525 \mathrm{psi}$ followed by $625,750,875$, and 925 psi. Pressure is obtained from the parametric input by subtracting 1.00 and scaling by 250 , and has a resolution of 0.1 volt or $\pm 12.5 \mathrm{psi}$ despite two decimal places reserved for parametric data in the instrument output.

Acoustic emission was monitored at all but the highest pressure for the full five minutes. The maximum pressure was only reached momentarily before the pressurization pump tripped. When disagreement among pressure gauge readings was noted, the test was halted for a period of time and the pressure dropped to about 800 psi. Pressure was then raised to 850 psi and held, and to 900 psi where it was maintained for 30 minutes. Acoustic emission was obtained during these later test periods, but because these pressures had already been exceeded, the primary utility of these emissions is as an indication of background noise level.

\subsection{Instrument Settings}

During testing, the event detection thresholds were set to $55 \mathrm{~dB}$ for all acoustic emission channels. This threshold level was observed to be between 3 and $8 \mathrm{~dB}$ above hard electronic background depending on the channel, and between about 1 and $4 \mathrm{~dB}$ above the soft electronic background. Here, the hard background level is the highest threshold where the event indicator light on the front panel of the instrument appears continuously lit, while the soft background level is the highest threshold level where sporadic background noise is detected at a rate judged to be sufficient to impede analysis. Hard background levels were typically found to be $50 \mathrm{~dB}$, a value of $47 \mathrm{~dB}$ for channels 1,6 , and 10 , and $51 \mathrm{~dB}$ for channel 7 . There does not appear to be a strong correlation between background level and sensor response. 


\subsection{Test Results}

\subsection{General}

The sum of acoustic emission events detected by each significantly active channel are plotted versus time in Figure 6, each with the superimposed pressure curve. Suspension of testing for water pumpdown is indicated by superimposed rectangles in the figure. Elimination of identified noise was performed prior to final analysis of the data. The criteria used for identifying noise signals is discussed in detail in a later section.

Detected events were insufficiently energetic to trigger more than one sensor in an array, thus only zonal location could be performed. Channels $1,4,7,9$, and 10 showed significant acoustic emission levels during the course of the test, while only sporadic emissions were observed on the remaining channels. No signals were detected by channel 5. Events detected on channels 1, 4, and 7 are apparently independent as indicated by the typically large and variable time intervals between events detected on different channels. There is an apparent correlation between events detected on channels 9 and 10 which is discussed below.

\subsection{Description of Acoustic Emissions}

\subsubsection{Channel 1}

Acoustic emission data from channel 1 shows continuous activity from the initiation of monitoring. As indicated in Figure 6 the emission rate is fairly constant before about 35 minutes, corresponding to a point just following pressurization from 400 to $525 \mathrm{psi}$, and is fairly constant again after about 51 minutes when the pressure is 750 psi and greater. The event rate during these periods fluctuates somewhat, decreasing from about 9 or 10 events/minute during 100 psi static pressure to about 6 to 6.5 events per minute until the end of the 400 psi static pressure period. The rate is about 7.5 events per minute in the interval from $55^{\circ}$ minutes to the end of the illustrated portion of the test. Measurements made during the 30 minute hold at 900 psi after the pressure gauge problem yielded a mean event rate of about 6 events per minute.

A significant increase in the emission rate occurs just following the pressure increase from 400 to $525 \mathrm{psi}$, (passing through the current operational pressure), and continues at a comparatively high rate with some tendency to taper off prior to a break in monitoring required to lower the water level at 625 psi. Following resumption of testing a very high emission rate occurs in conjunction with a pressure increase from 625 to 750 psi. Following stabilization of the pressure at $750 \mathrm{psi}$, the emission rate decreases to approach that seen prior to the $400-525$ psi pressurization and remains at this level. 

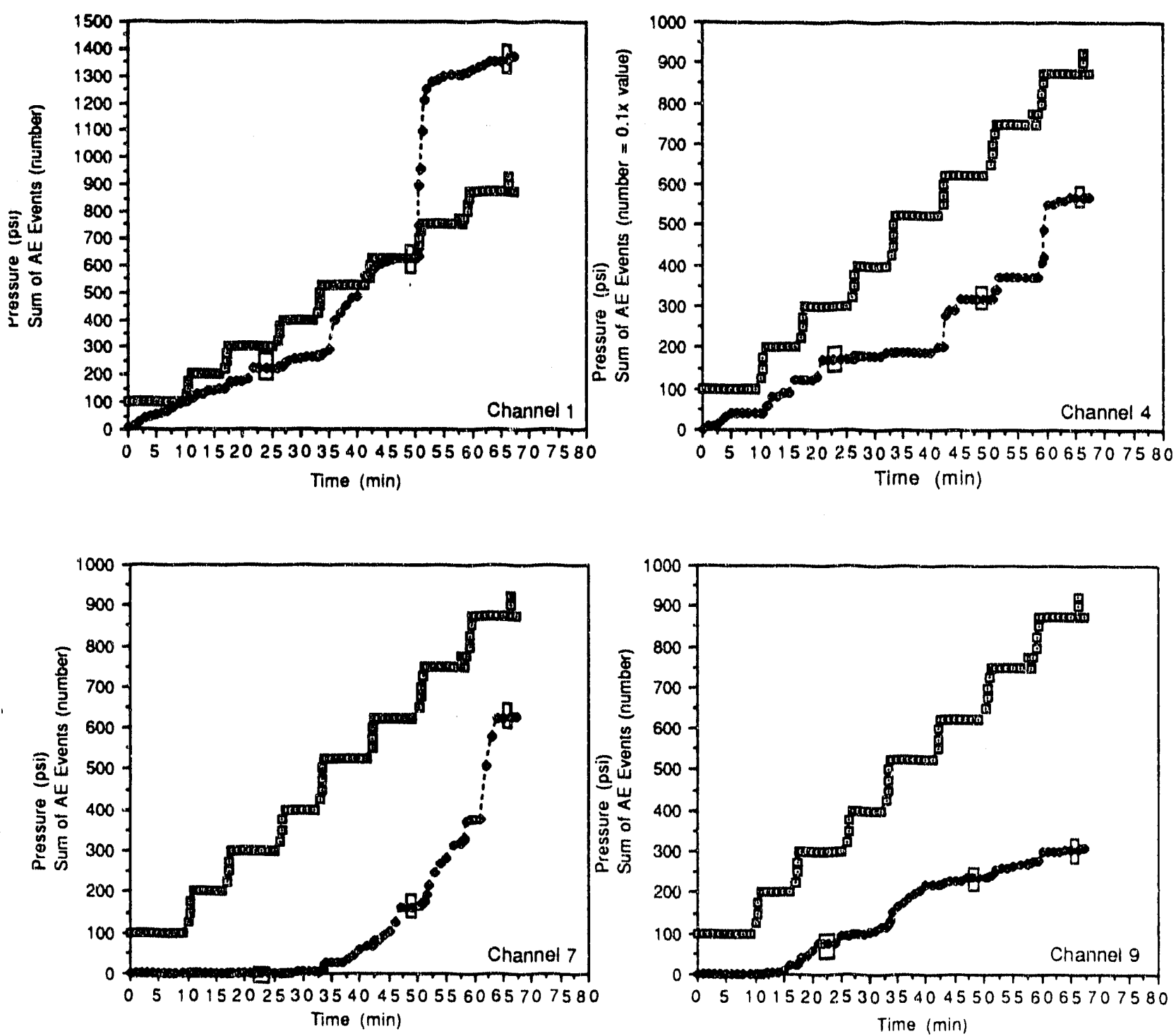

Figure 6. Cumulative Acoustic Emission Events and Applied Pressure as a Function of Time for All Active Channels

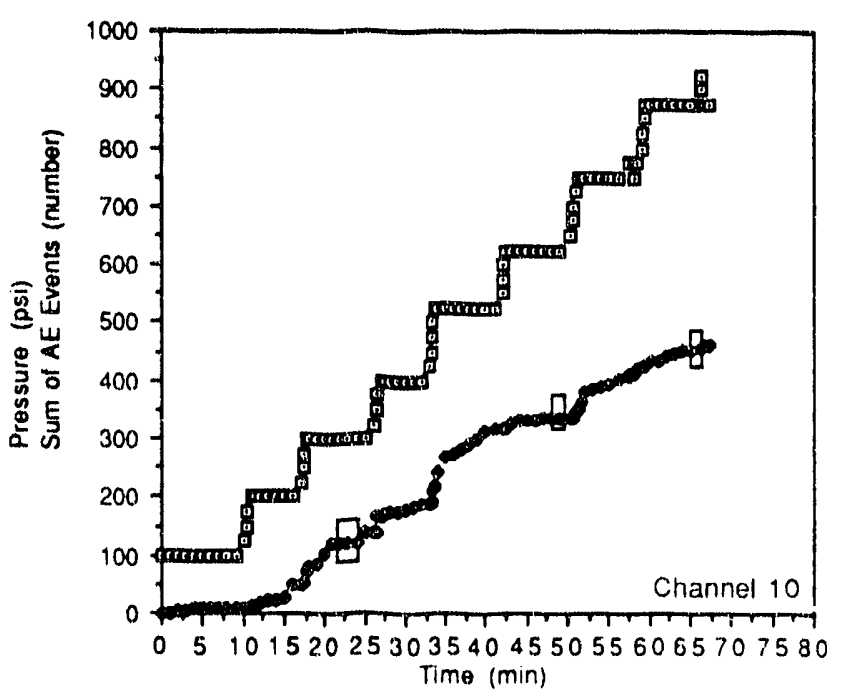


Acoustic emission amplitudes over the entire test range from 57 to $78 \mathrm{~dB}$. These signals can be characterized as high amplitude, short duration events with rise times typically greater than or equal to the duration. Possible trends are identifiable by calculating and comparing the statistical distribution of amplitudes over different regions of the test data. For example, during the first ten minutes of testing with the pressure at $100 \mathrm{psi}$, the most likely amplitude is found to be $60 \mathrm{~dB}$. The probability of this measurement is only $12 \%$ due the spread of the data which here ranges from 57 to $76 \mathrm{~dB}$. The spread also accounts for the mean amplitude value during this period being $\mu=66.5 \mathrm{~dB}$ with a standard deviation of 5.7. This may be compared with the more active period when the pressure was being increased to $750 \mathrm{psi}$ and the event rate was about 100 events/minute. In this region the most likely amplitude is $67 \mathrm{~dB}$ with probability of $24 \%$. The amplitudes here fall into a more restricted range of 58 to $72 \mathrm{~dB}$. The mean amplitude is $\mu=64.5 \mathrm{~dB}$, lower than in the region previously described, and the standard deviation is $4.6 \mathrm{~dB}$. These results and their implications are discussed below.

\subsubsection{Channel 4}

Far less activity was seen on channel 4, where only 53 events were detected over the entire test, with a maximum rate of 16 events per minute. The event rate is extremely low except during transient pressurization beginning at the 525 to 625 psi transition. An examination of the amplitude distribution shows a range from 56 to $68 \mathrm{~dB}$, and the most likely amplitude is $58 \mathrm{~dB}$ with a probability of $30 \%$. The higher amplitude events cause the mean amplitude value to be $62 \mathrm{~dB}$ with a standard deviation of $3.7 \mathrm{~dB}$. A sufficient number of events occurred during pressurization from 750 to 825 psi that analysis of amplitudes in this region may be informative. The first event occurring during pressurization has an amplitude of $68 \mathrm{~dB}$ and is followed by a number of events with amplitudes of 56 and $58 \mathrm{~dB}$. The most likely amplitude in this region is again $58 \mathrm{~dB}$ with a likelihood of $31 \%$. The mean is $60.6 \mathrm{~dB}$ with standard deviation of $3.5 \mathrm{~dB}$.

\subsubsection{Channel 7}

Except for a pair of events following pressurization to 200 psi, channel 7 was quiet until the pressure transition from 400 to 525 psi. The few events accompanying this transition were followed, after some delay, by a substantial increase in the event rate. Monitoring during this period was interrupted by a break in the test to pump down the water level, but the high rate was still detected after monitoring was resumed. A pressure increase from 750 to $875 \mathrm{psi}$ also appears to initiate a substantial event rate increase. No significant change in the emission rate accompanied the short duration pressure increase to the maximum pressure of 925 psi.

Channel 7 event amplitudes range from $53 \mathrm{~dB}$ to $65 \mathrm{~dB}$. The presence of $53 \mathrm{~dB}$ events is not a concern despite the nominal $55 \mathrm{~dB}$ threshold for reasons discussed below. The 
DAT-\# DDD HH:MM:SS. mmmLILILIN FARAI FI/CC CH SEQ-\# DUFAT. COUNT ENFIGY AMF F

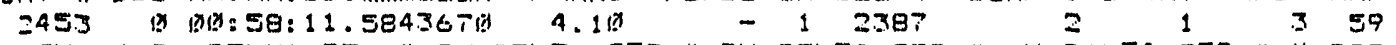
EVENT \# CH DELTA SEQ-\# CH DELTA SEQ-\# CH DELTA SEQ-\# CH DELTA SEQ-\# $X$ F.OS Y

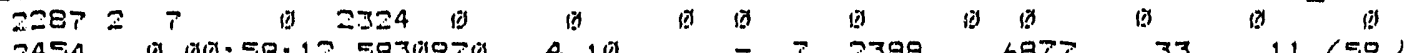

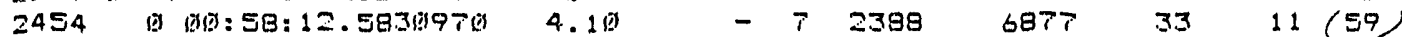
EVENT \# CH DELTA SEQ-\# CH DELTA SEQ-\# CH DELTA SEQ-\# CH DELTA SEQ-\# X_FOS Y_

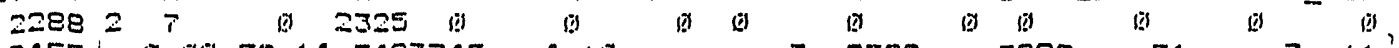

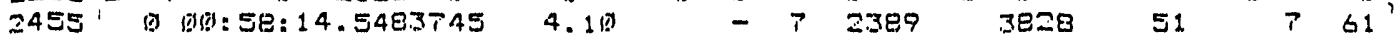
SUENT \# CH DELTA SEQ-\# CH DELTA SEQ-\# CH DELTA SEQ-\# CH DELTA SEQ-\# X...ES Y

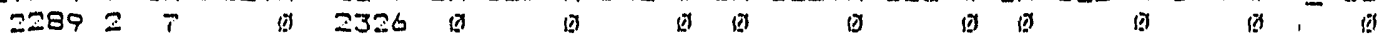

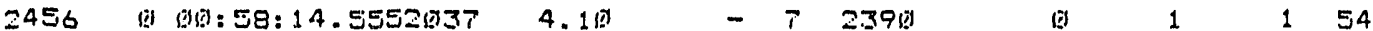
EVENT \# CH DELTA SEQ-\# CH DELTA SEQ-\# CH DELTA SEQ-\# CH DELTA SEQ-\# X_FOS Y _

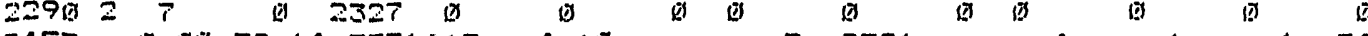

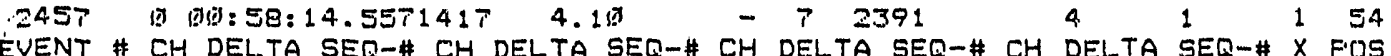
EVENT \# CH DELTA SEQ-\# CH DELTA SEQ-\# CH DELTA SEQ-\# CH DELTA SEQ-\# X_FUS Y_

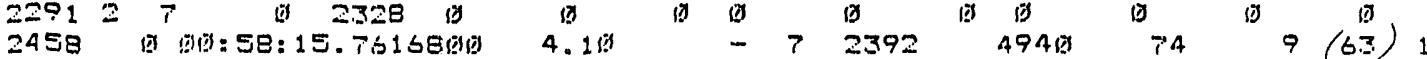

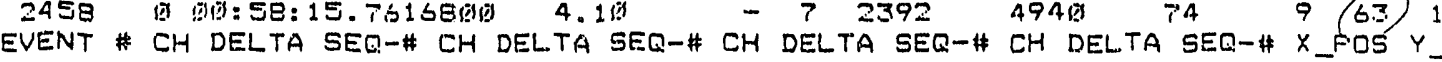
$\begin{array}{lllllllllllll}2292 & 2 & T & 0 & 2329 & 0 & 0 & 0 & 0 & 0 & 0 & 0 & 0\end{array}$ 'EVENT \# CH DELTA SEQ- \# CH DELTA SEQ-\# CH DELTA SEQ-\# CH DELTA SEQ-\# $\times$ FOS Y

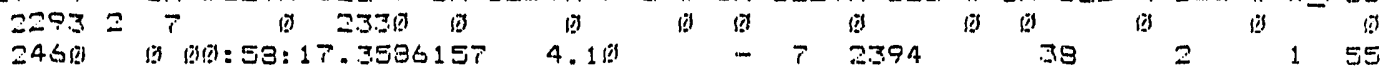
24S(3) EVENT \# CH DELTA SEQ-\# CH DELTA SEQ-\# CH DELTA SEQ-\$ CH DELTA SEQ-\# X FCS Y

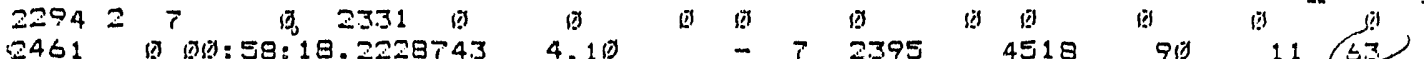
LVENT \# CH DELTA SEQ.-\# CH DELTA SEQ-\# CH DELTA SEQ-\# CH DEL.TA SEQ-\# X_FDS Y

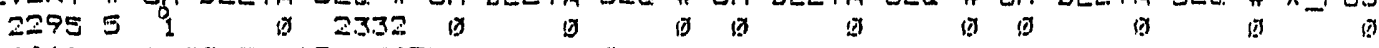
2452 (9):58:18.2343393 4.16 - 7.2396 GUENT \# CH DELTA SEQ-\# CH DELTA SEQ-\# CH DELTA SEQ-\# CH DELTA SEQ-\#. FOS Y.

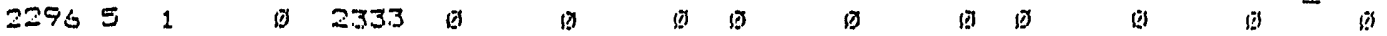

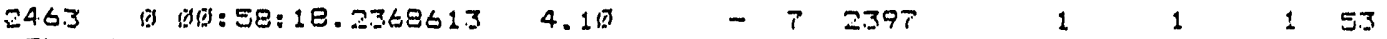

EVENT \# CH DELTA SEQ-\# CH DELTA SEQ-\# CH DELTA SEQ-\# CH CELTA SEQ - X X FOS Y $229727 \quad$ i 23.34 (j) 2464 D $63: 58: 19.5671845 \quad 4.10 \quad-\quad 7.2393 \quad 5594 \quad 106 \quad 10 \quad 54 \quad 1$ EUENT \# CH DELTA SEO-\# CH DELTA SEQ-\# CH DELTA SEQ-\# CH DELTA EEQ-

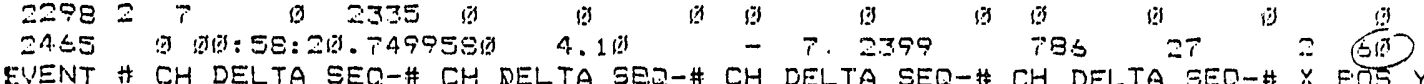

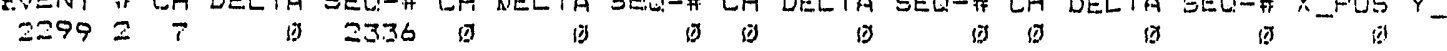

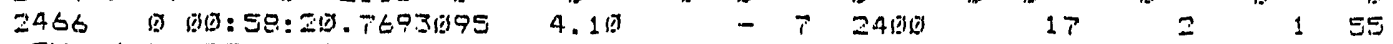
E'VENT \# CH DELTA SEQ-\# EH DELTA SEQ-\# CH DELTA SEQ-\# CH DELTA SER-\# X_FOS Y

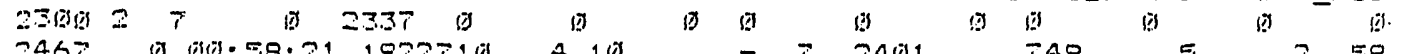

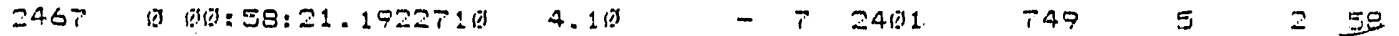
EVENT \# CH DELTA SEQ-\# CH DELTA SEQ-\# CH DELTA SEQ-\# CH DELTA SEQ- \# X FCS Y _ $23_{1}=T \quad$ () 2338 is 2468 10 EVENT \# CH DELTA SEQ-\# CH DELTA SEQ-\# CH DELTA SEQ-\# CH DELTA SEQ-\# X_FOS Y _

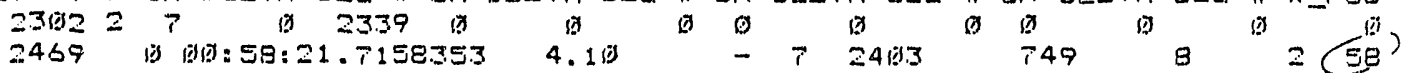
EVENT \# CH DELTA SEQ-\# CH DELTA SEQ-\# CH DELTA SEQ-\# CH DELTA SEQ-\# X FOS Y

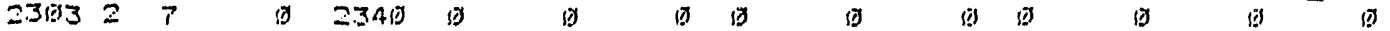

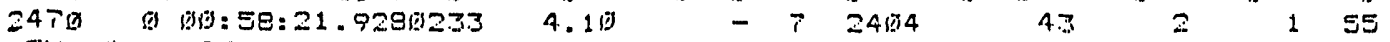
EVENT \# C.H DELTA SEQ-\# CH DELTA SEQ-\# CH DELTA SEQ-\# CH DELTA SEQP-\# X_FOS Y _

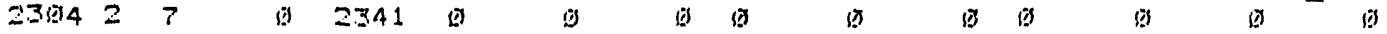

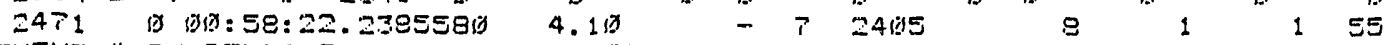
EVENT \# CH DELTA SEQ-\# CH DELTA SEQ-\# CH DELTA SEQ-\# CH DELTA SEQ-M X_FOS Y

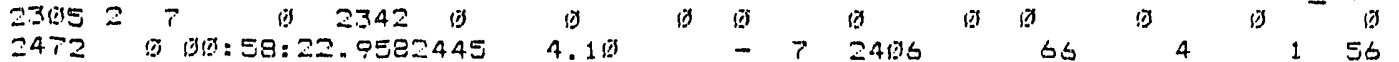
2472 OVET \#

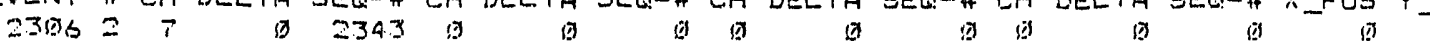

Figure 7. Sample of Raw Data Obtained During High Channel 7 Event Rate 


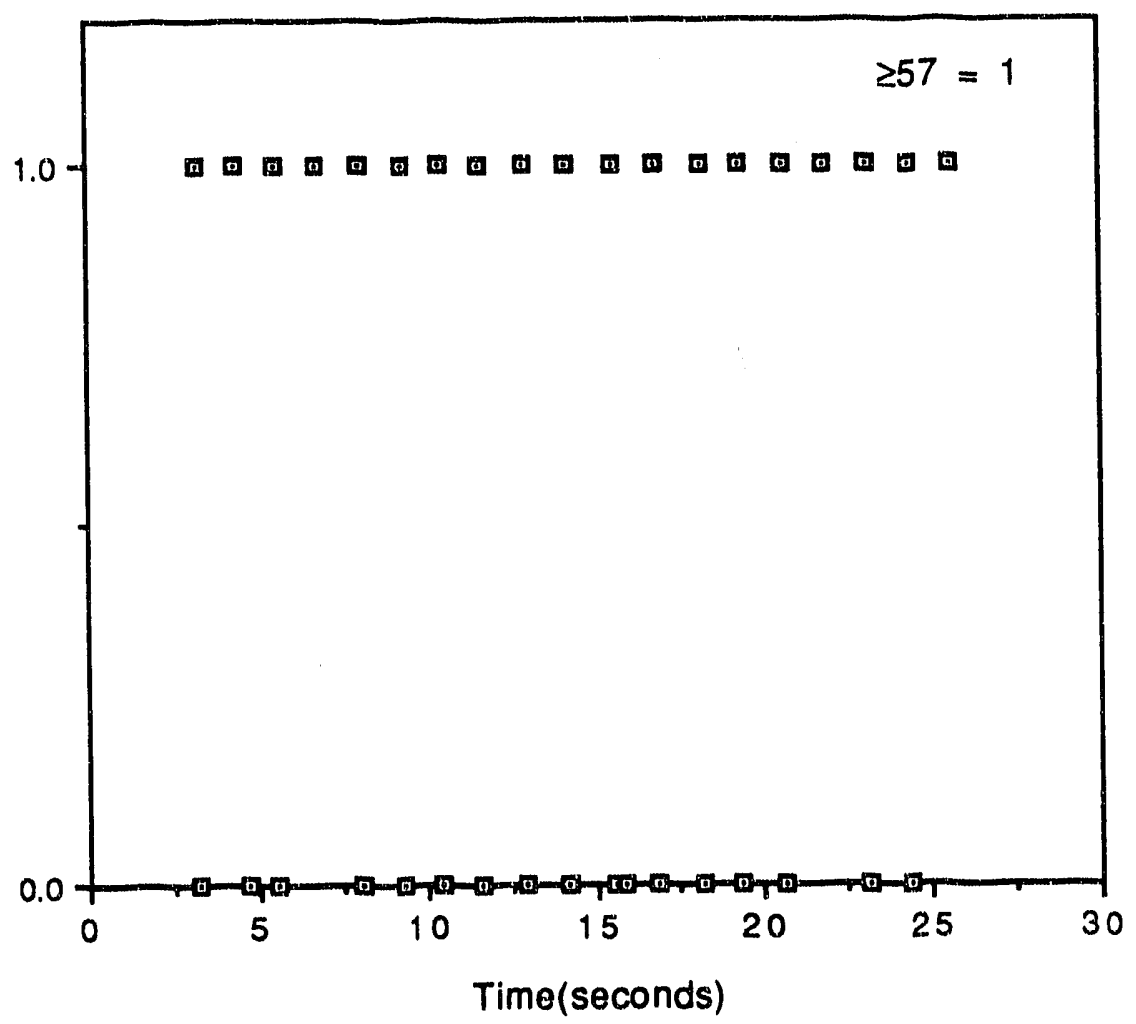

Figure 8. Illustration of the Periodicity in Channel 7 Events. Events with amplitude greater than 57 decibels are assigned the value 1 , while the remainder are assigned the value zero. The ordinate is the time of occurrence of the events relative to a convenient origin.

examination of signal amplitudes and event times from various regions of the test data indicates, however, that many or all higher-amplitude events may be caused by noise unrelated to damage. Beginning near 58 minutes into the test (near the end of the 750 psi hold period) and continuing for about 15 minutes, a number of events were found with amplitudes in excess of $57 \mathrm{~dB}$. A sample of raw data from this region is reproduced in Figure 7. These events occurred periodically at intervals of about $1.2-1.3$ seconds initially, and were interspersed with between zero and five lower amplitude events. The interval between these higher amplitude events increased in a fairly regular manner while their amplitudes decreased until the events were no longer distinguishable. To illustrate the periodicity of these high amplitude data, a series of representative sequential events were plotted in Figure 8 with time as the abscissa, and with ordinate value 1 if the amplitude was $57 \mathrm{~dB}$ or greater and value zero otherwise. All of these data were removed prior to final analysis. Of note is that similar data were seldom observed outside of the region described above. 
The time delay between a high amplitude event and each of the following lower amplitude signals was next examined and listed in conjunction with the duration of the larger signal. The same delays were found to occur repeatedly and corresponded fairly well with delays expected from a sensor effect as discussed below. These signals were therefore also discarded with some confidence that they are not of interest. A conservative estimate of the valid data accumulation, retaining most events whose origin is uncertain, is illustrated in Figure 9.

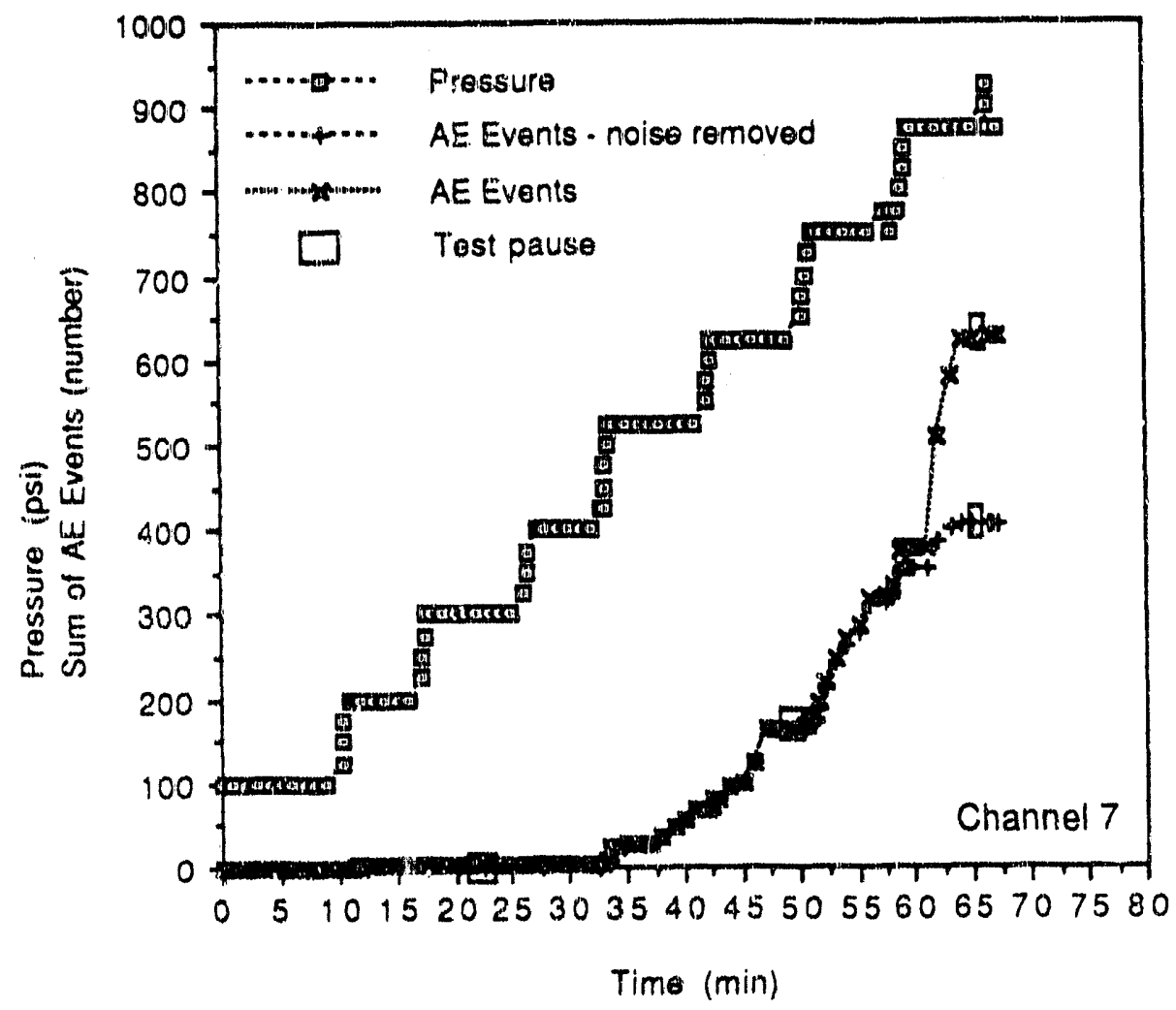

Figure 9. Channel 7 Data with Periodic and Internal Reflection Events Removed Overlayed on the Unfiltered Data shown in Figure 6

Prior to this period, the most likely amplitude is $54 \mathrm{~dB}$ with $38 \%$ likelihood, a mean amplitude of $55.5 \mathrm{~dB}$, and deviation $\mathrm{s}=1.9 \mathrm{~dB}$. The distribution is therefore fairly narrow compared to the channel 1 distributions described earlier. Since the peak of the distribution lies above the minimum amplitude, most of the emissions caused by this source were probably detected. 


\subsubsection{Channels 9 and 10}

In contrast to general isolation of detected events observed on all other channels, those detected by channels 9 and 10 appear to be correlated. This correlation is apparent in the similarity of the plots of events versus time for these two channels shown in Figure 6. A large number of events are detected first by channel 9 , followed by orie or more channel 10 events delayed by about 4.4 -4.9 milliseconds. Assurning first that channel 9 and 10 detect the same propagating signal, these large delta-time values cause the wave speed closest to a reasonable value to be obtained using the maximum acoustic path between the two sensors. This maximum path length is about 27 inches, which yields an effective wave speed of $.006 \mathrm{in} . / \mathrm{sec}$. or so, one-tenth that of water. This suggests either that substantial dispersion of the signal occurs as it travels from sensor 9 to 10 , causing the event detector on the two channels to trigger on entirely different parts of the signal, or that the channels are cor pled mechanically or electronically and that all or part of the data is noise. The difference in amplitudes between the channel 9 and channel 10 response for correlated events ranges from 8 to $13 \mathrm{~dB}$, not inconsistent with a highly dispersed and/or attenuated signal whose source is closer to sensor 9 (the top of HB-4). Examination of the amplitudes shows those of channel 9 to range from $57 \mathrm{~dB}$ to $71 \mathrm{~dB}$, while channel 10 amplitudes range from $49 \mathrm{~dB}$ to $53 \mathrm{~dB}$. The channel 10 events therefore are well below the set threshold, indicating an error condition. In the laboratory the instrument was examined for the possible source of this condition. Re-loading the program setup in the SPARTAN and generating controlled input signals using a simulator showed that the threshold was in fact set to $55 \mathrm{~dB}$ during testing as intended, and that the minimum recorded signal amplitude should be $60 \mathrm{~dB}$ due to lack of proper calibration. It is likely therefore that there is a condition internal to the instrument which causes spurious below-threshold events to occur. This condition has been noted in an instrument by a different manufacturer used for a number of years at PNL and on which the SPARTAN technology is partially based. The number of pseudo-events generated internally by this other instrument also outnumbered the valid events as observed in the present case with the SPARTAN data. In any case, if channel 10 data is valid and due to crack extension, the only information lost by rejection of the data is a possible enhanced definition in the source location. As shown below, channel 10 is badly out of calibration near the sensor resonance frequency, requiring the actual threshold value to be about $44 \mathrm{~dB}$ if the channel 10 events are valid.

Although this channel is out of calibration, the offset in recorded amplitude should not affect comparative analysis. Significant emissions detected by channel 9 first appear during static pressure at 200 psi. The event rate decreases toward the end of this region in the 400 psi staric pressure interval. In this early portion of the test the amplitude range is 58 to $68 \mathrm{~dB}$, the most likely amplitude is $60 \mathrm{~dB}$ with a likelihood of $30.3 \%$, a mean of $63.0 \mathrm{~dB}$, and deviation of $3.3 \mathrm{~dB}$. Higher amplitude events in this region occur predominately within the first few minutes. The pressure increase from 400 to 525 psi. is accompanied by a second rise in the acoustic emission event rate. Although the amplitude statistics are similar, the initial amplitudes are low, and high amplitude events 
occur sporadically throughout the measurement interval. These events have a range of 57 to $71 \mathrm{~dB}$ (the increase possibly due to a larger number of samples), a most likely value of $60 \mathrm{~dB}$ with $23.2 \%$ likelihood, and $\mu=63.2 \mathrm{~dB}$ with $s=4.2 \mathrm{~dB}$.

Late events detected during repressurization after establishing correct pressure readings and the 30 minute static pressure period at $900 \mathrm{psi}$ occurred in two groups. The first consisted of seven events occurring nearly simultaneously just after pressure was raised from 800 to 850 psi. Delta-times indicate that a portion of these events may be noise. Three distinct events also occurred at 900 psi over a period of about five minutes.

\subsection{Evaluation of Test Results}

No more than one sensor detected any given event. This conclusion results from the observation that the SPARTAN detected only one event validated by an array during the entire test, and also from direct examination of the arrival time differences and hit sequences of the test data. During pulser tests, surface waves and water-borne signals were detectable on multiple sensors and often without substantial amplitude differences. Detection of an event by only one of a pair of closely spaced sensors, such as the pairs 2 and 3, 4 and 5, 6 and 7, and 9 and 10, therefore indicates that the source a) was noise originating in the sensor electronics, or b) originated in the vessel wall in the near vicinity of the active sensor. Electronic noise as an option can be eliminated since such noise would probably not exhibit the observed correlation with pressure. Sensors 1 and 8 are relatively isolated, hence a signal originating at some distance from the sensor may be detected on one of these channels but be undetected elsewhere because of dispersion and attenuation effects.

\subsection{Channel 1}

Channel 1 background acoustic emission does not exceed about 6 events per minute, which is the lowest level measured on this channel during testing. The background rate was measured at 900 psi, but due to having achieved higher pressure during an earlier interval, sources such as crack extension which are subject to the Kaiser effect ${ }^{1}$ would be eliminated. Sources subject to the felicity effect ${ }^{2}$ are unlikely to remain active for an extended period at constant pressure, while the observed emission rate at $900 \mathrm{psi}$

\footnotetext{
${ }^{1}$ Sources subject to the Kaiser effect are not activated until the previous maximum applied stress is exceeded.

${ }^{2}$ The felicity effect describes non-Kaiser effect sources.
} 
appeared to be about constant. Contributions to the background rate occurring at 400 psi and below probably include innocuous sources such as dripping water, or possibly the motion of components loosened by lowered pressure. Contributing to the rate at $750 \mathrm{psi}$ and above may be emissions from the source active during the preceding high emission rate interval continuing at a lower level of activity. No signals could be identified as noise with confidence based on signal characteristics, hence the entire data set was retained for analysis.

The amplitude distributions revealed two possibly significant trends. First, the range of amplitudes occurring in various test regions were found to vary via the presence or absence of amplitudes above about $70 \mathrm{~dB}$, and the most likely value was also observed to fluctuate. The variations in these two parameters show some interdependence and also dependence on test conditions. The principle trends are that a large amplitude range (58 $-76 \mathrm{~dB}$ or greater) combined with a low most- likely amplitude of $60 \mathrm{~dB}$ occur when the emission rates are close to background. The regions with high most-likely amplitudes appear to accompany high emission rates. This latter increase is probably not due entirely to the superposition of nearly simultaneous events, since the highest value of 71 $\mathrm{dB}$ does not accompany the highest emission rate. Further examination of these relationships is probably not profitable at present since it does not appear to help identify the source process.

Channel 1 is located near the pressure vessel head, and it is easy to rationalize the observed emission activity in terms of any one of a number of possibilities. Crack growth cannot be ruled out, since the delayed increase in activity following the transition through the operating pressure is consistent with this source, as is the subsequent high emission rate tapering off as stress is relieved, as the crack front is pinned, or perhaps as the micro-crack mechanism changes. In the first two cases an increase in pressure may re-activate the crack. Similar behavior has been seen during stress-corrosion cracking in iron alloys and stainless steel in small laboratory specimens subjected to step-and-hold loading [3].

The background emissions present throughout the test may also indicate leakage. Because of single sensor validation and insufficient knowledge of the overall condition of the vessel, however, any suggestion remains highly speculative.

\subsection{Channel 4}

The apparent correlation between the small number of detected acoustic emissions and the pressure transitions is similar to that obtainable from a stationary crack subject to step loading or plastic deformation in regions of high localized stress, but may also be caused by structural interfaces which exhibit relative motion during load changes. The distribution of the event amplitudes indicates that most events produced by the source were detected. This can be interpreted as a low level of source activity. 


\subsection{Channel 7}

Although the variability of amplitude range and most likely amplitude observed in channel 1 data appears to be absent from channel 7 once noise is removed, crack growth again cannot be ruled out as a possible source mechanism. The amplitude consistency (disregarding noise) observed here may be caused by the absence of signals from a secondary source possibly present in channel 1 data. It is evident that the channel 7 source is activated or amplified by an increase in pressure past the current operating pressure, and for reasons discussed elsewhere, the source is probably located within the pressure vessel wall in the vicinity of the channel 7 sensor attachment point.

Once begun, source activity increases almost sigmoidally, showing little direct dependence on the rate of pressure change. In an active crack, this type of emission may accompany crack advance in the absence of a change in mode or increment size over much of the range of applied stress. A $\mu$ crack would also not necessarily experience sufficient stress-relief through extension to cease growing over the time interval between pressure steps. In this case, the apparent roll-off in acoustic emission rate at high pressure may have one of two interpretations: although fewer enissions may occur if the crack growth rate decreases, the same decrease in emission rate has been observed in stainless steel during stress corrosion cracking tests in the laboratory when a crack becomes unstable. This latter phenomenon is caused by a drop in the energy released per acoustic emission event, possibly coupled with an increase in crack increment size per acoustic emission event [3]. The same caveat regarding speculative source identification, however, also holds for channel 7 data.

\subsection{Channels 9 and 10}

Due to the Kaiser effect, the most likely crack-related mechanism which could cause the initiation of emissions at less than the operating pressure is probably crack interface noise caused by contact between crack faces at low pressure. Oxide rupture is not likely unless the oxide was formed on an external surface which is, however, clad with stainless steel. The initial high amplitude events in the first active region indicate a source which had stabilized below 200 psi and required some three or four minutes at this pressure to activate. The second active region beginning during the 400 to 525 psi transition may be a separate mechanism due to the temporal distribution of higher amplitude events. As in other cases, the behavior expected from a crack growth source mechanism does not conflict with the observed data.

Leakage is also a possible source, and the same observations made in reference to the channel 1 evaluation above apply to channel 9 data. 


\subsection{Discussion}

Although AE generally behaved in a manner that is not inconsistent with crack growth emission, other sources may be activated by similar stimulation. The information available for analysis from acoustic emission monitoring consists primarily of whether acoustic emissions were detected and on which channel or channels, the long-term behavior of acoustic emissions, the relationship of the emissions to pressurization, and the relative cistributions of the signal characteristics. Source location using triangulation could not be performed since multi-sensor detection of single events did not occur. This may be interpreted as an indication that all detected signals are noise, but high ultrasonic attenuation in stainless steel and other effects may also primary contributors to this result as discussed in Section 12.4.

Detected emissions should first be examined for noise, and such signals removed from the data. Normally, the most reliable method of identifying crack growth and removing noise in the field is source location. If source locations are narrowly distributed near a high-risk region or if locations tend to progress along or through such a region, the likelihood of a crack increases. Each event can be verified if triangulation-type source location can be performed, and sources with uninteresting point-of-origin eliminated. In the present case only zone-location was feasible, which prevents both high accuracy and event-by-event verification.

Correlations between acoustic emissions and pressure are suggestive, but appear somewhat ambiguous. The components of a structure tend to adapt stable inter-relationships at standard operating conditions which may become unstable when the conditions are changed. Although installed gaskets, for example, which are compressed from continued loading permit relative motion of the interfaces when the load is removed, such emissions should cease at higher loads. If there are no such local sources but there are local high-risk regions, the likelihood of crack growth becomes greater.

Statistical analysis of signal parameters aids in identifying noise, identifying the presence of multiple sources, recognizing a change in the behavior of a source, and determining what fraction of individual source processes (e.g., crack increments) were detected. Of those characteristics provided by the SPARTAN, counts and energy are ambivalent because of their dependence on two or more other signal characteristics, and did not appear to provide significant information during initial cursory examination. The characteristic which appeared to provide the greatest amount of information was the signal amplitude, which aided in identifying noise in channels 10 and 7 , and was a useful indicator of source behavior as described in the relevant sections above.

Some comparison was made to the acoustic emission behavior of laboratory specimens during stress-corrosion cracking under step-and-hold loading. Similarities in behavior are 
not intended to be proof or necessarily strong indicators of the presence of growing cracks in the HFIR vessel, but do provide some measure of supportive evidence.

Leakage acoustic emission is typically continuous type caused by turbulent fluid flow through an orifice, similar in appearance to and additive with electronic background noise. A transient detector such as the SPARTAN would detect a leak of the appropriate size as a series of sporadic transients. Gas leakage studied in the laboratory produced acoustic emissions with RMS voltage proportional to the flow rate, where the flow rate in these laboratory tests was varied by changing the diameter of a circular orifice while maintaining a constant pressure gradient. Change in pressure through a constant diameter circular orifice was observed to cause a similar change in acoustic emission RMS voltage A change in the RMS voltage would produce a corresponding change in the event rate detected by a transient monitor, limited by the instrument reset time and its method of accommodating events with durations exceeding a maximum allowed value. Event rates did not increase in channel 1 and channel 9 data in the manner expected for the simplest case of leakage- induced emissions, but other phenomenon effecting the signals cannot be ruled out. A change in flow conditions or stabilization of the flow rate because of friction and fluid viscosity would change or stabilize the acoustic emission level. An orifice may become clogged with debris, or open or close as pressure is increased. Leak detection capability has also been somewhat compromised by the test conditions. Because detection thresholds were established at pressure, leaks existing at 100 psi may be undetectable unless their emission increases later in the test.

\subsection{Noise and Instrumentation Effects}

Noise refers to any detectable signal other than those caused by the source of interest. The two general categories of noise are:

a) Active sources other than the source of interest detected as acoustic emission by the sensor,

b) Electrical noise either detected by or produced in the acoustic emission instrument. Noise of the former type would include the effects of electrically dirty power lines, cross-talk between sensor leads, and RF radiation. Noise of the latter type may be caused by failing circuit components or poor connections.

Noise may be identified with more-or-less effectiveness by a number of means. Signals caused by type A noise typically have reasonable signal parameters, which often deviate from values expected for the primary source. As an example, noise caused by rubbing between two adjacent components such as a joint pin and a bracket may exhibit "Yceptionally long rise times and durations compared to emissions from crack growth. Type B noise also exhibits unusual characteristics, often notable because sensor effects 
are absent. This latter type of noise may be very low or very high frequency or very short duration. Electrical noise has also been observed which occurs as groups of signals of identical or smoothly varying amplitude, and with individual events in the group separated by constant or progressive time intervals. Individual events separated by a constant time interval is a reliable indicator of noise, since emission sources related to damage rarely produce sustained highly periodic emissions.

\subsection{Instrumentation Effects on Acoustic Emissions}

Certain data was eliminated from the data set because of the likelihood that it is noise. As stated above, channel 10 events were ignored because most exhibited amplitudes are well below the detection threshold, and appeared correlated with channel 9 events. In order to confirm that these signals could not arise from valid data, simulated acoustic emission signals were used to trigger the SPARTAN and the response of the instrumerit examined. An acoustic emission simulator manufactured by Acoustic Emission Associates produces transient signals with adjustable amplitude, rise and decay times, and adjustable frequency. The SPARTAN was initialized using the software routine created for the HFIR vessel tests. Examination of the threshold settings verified that each channel was set at $55 \mathrm{~dB}$ with zero internal gain. The simulator was set to produce a signal at $250 \mathrm{kHz}$ (somewhat below the nominal $350 \mathrm{kHz}$ center frequency of the sensors), with a rise time of about $10 \mu$ seconds, initial amplitude of $55 \mathrm{~dB}$, and a decay rate of $50 \mathrm{~dB} / \mathrm{msec}$. Signals with these characteristics were used for all tests described below except where noted. The simulator amplitudes and other characteristics were confirmed by examination of signals on a Nicolet 4094A digital oscilloscope, which showed a range of positive peak amplitudes of 51.2 to $56.0 \mathrm{mV}$ with $\mu=53.2 \mathrm{mV}$, s $=$ $1.4 \mathrm{mV}$, and negative amplitudes from 54.4 to $59.1 \mathrm{mV}$ with $\mu=56.2 \mathrm{mV}$ and $\mathrm{s}=1.5$ $\mathrm{mV}$ where $\mathrm{s}$ is the root variance for small data sets, calculated using $1 /(\mathrm{N}-1)$ rather than $1 / N$. Using the standard formula

$$
\text { decibels }=20 \log \left(\frac{V_{2}}{V_{1}}\right)
$$

the reference voltage $V_{1}$ is found to be $0.1 \mathrm{mV}$, confirmed by similar measurements using simulator amplitudes of $60,70,80$, and $90 \mathrm{~dB}$. The millivolt values measured from the oscilloscope therefore range from 54.2 to $55.4 \mathrm{~dB}$, thus for a nominal $55 \mathrm{~dB}$ simulator signal, 54 or $55 \mathrm{~dB}$ amplitudes are expected to be displayed by the SPARTAN.

All $55 \mathrm{~dB}$ input signals to channel 10 were detected using the input signal described above, although measured amplitudes displayed by the SPARTAN were $60 \mathrm{~dB}$ for these inputs. The data acquisition instrument also detected $54 \mathrm{~dB}$ input signals displayed as 58 or $59 \mathrm{~dB}$, but was unable to detect $53 \mathrm{~dB}$ inputs. The tests were re-run with an input 
signal frequency of $100 \mathrm{kHz}$, which yielded $55 \mathrm{~dB}$ measured for $55 \mathrm{~dB}$ input amplitudes, and $53 \mathrm{~dB}$ measured for $54 \mathrm{~dB}$ inputs. Other measurements at various frequencies indicate that the observed results are due to the shape of a frequency filter on each channel internal to the SPARTAN which produces $5 \mathrm{~dB}$ or so of signal amplification near the filter's center frequency, with a low-end roll-off to zero gain near $100 \mathrm{kHz}$. The threshold detector follows the filter in processing order. In no instance were signals with amplitudes below $53 \mathrm{~dB}$ detected in the laboratory on channel 10 , indicating that signals seen during the test are probably internally-generated noise.

Each remaining channel was similarly examined. Results show not only the effects of the filter, but also indicate that other channels were also out of calibration. Results are summarized below. It should be noted that the center frequency on each sensor varied somewhat, therefore data in Table 2 should be taken as an estimate only. All channels have zero internal (user adjustable) gain and are set to a $55 \mathrm{~dB}$ threshold:

\section{Table 2. Minimum Detectable Input Signal Amplitudes and Range of Displayed Amplitudes}

The nominal threshold is $55 \mathrm{~dB}$ for all channels; input signal frequency is $250 \mathrm{kHz}$.

\begin{tabular}{|c|c|c|}
\hline Channel & $\begin{array}{c}\text { Minimum Detectable Input } \\
\text { Signal Amplitude (dB) }\end{array}$ & $\begin{array}{c}\text { Value or Range of Measured } \\
\text { Amplitudes at the Minimum } \\
\text { Detectable Input Signal Level (dB) }\end{array}$ \\
\hline 1 & $55^{*}$ & $55-56$ \\
\hline 2 & 58 & $56-58$ \\
\hline 3 & $56^{*}$ & 55 \\
\hline 4 & $55^{*}$ & 56 \\
\hline 5 & 58 & $57-60$ \\
\hline 6 & $57^{*}$ & $57-58$ \\
\hline 7 & $52^{*}$ & 53 \\
\hline 8 & $55 \dagger$ & 56 \\
\hline 9 & 54 & 58 \\
\hline 10 & $55^{*}$ & 60 \\
\hline
\end{tabular}

*Usually less than one in ten of the signals were detected at one $\mathrm{dB}$ lower input signal amplitude. tA small fraction of events at one and two $\mathrm{dB}$ lower input signal amplitude were detected. 
Other parameters obtained from the input signal were compared with measurements by the SPARTAN. Using a $55 \mathrm{~dB}$ input signal, rise times measured from the oscilloscope display ranged from roughly 5 to $15 \mu$ seconds. SPARTAN-measured values ranged from 25 to $32 \mu$ seconds, not unreasonable for simulated signals converted to dB, since small amplitude differences between consecutive peaks in uniformly decaying signals may be lost by the vertical compression accompanying a logarithmic transformation. Although signal duration is often shorter than the rise time both in the current validation tests and in pressure vessel test data, this is not proof of noise since duration appears to be measured from the time of the peak amplitude. Energy and counts were not used in data analysis and so were not examined.

\subsection{Peculiarities of the Waveguide Sensor Response}

A displacement spike detected by a waveguide sensor has been observed to convert primarily into two rod modes, one travelling with a longitudinal wave speed (referted to as the "longitudinal" mode here) followed by a second wave group travelling at about the shear wave speed (the "shear" mode). Both of these waves will be internally reflected, and may be detected as independent events if they retain sufficient amplitude upon returning to the sensor end of the waveguide. Using estimates of the wave speed in steel rod taken from laboratory data obtained during a previous program, the difference in arrival of the direct first and second modes should be about $820 \mu \mathrm{sec}$ or so if the event originates at the sensor tip. A list of the expected arrival times for consecutive internal reflections of the two modes is given below:

Table 3. Estimated Arrival Times for Internally Reflected Wavemodes in a $17^{\prime} 8^{\prime \prime}$ Waveguide Sensor

\begin{tabular}{|c|c|c|}
\hline Reflection & Longitudinal (msec) & Shear (msec) \\
\hline 0 & 0 & 0.82 \\
\hline 1 & 2.23 & 2.46 \\
\hline 2 & 4.46 & 4.10 \\
\hline 3 & 6.69 & 5.74 \\
\hline 4 & 8.92 & 7.38 \\
\hline 5 & 11.15 & 9.02 \\
\hline
\end{tabular}


The shear mode or some reflections may not be detected because of various pre-test settings of the analysis instrument. These settings are required to allow the analysis machine to define an event. The two settings pertinent to the present analysis are the time delay between consecutive threshold crossings and the system re-arm time. The former value is necessary because the oscillating signal continually crosses the threshold within a rising and falling envelop. If the signal remains below threshold for a sufficient amount of time, the event is ended and the system is re-armed to prepare for the next arrival. The time delay to determine the end of an event was set to 1.2 milliseconds, while the rearm time was set to 500 microseconds. Internal reflections will not be detected as new events unless the delay in the arrival of a reflected mode exceeds the rise time plus duration of the prior event plus 1.7 milliseconds.

High-amplitude periodic events in the channel 7 data suspected to be noise were followed by low-amplitude events havisig durations and rise times of typically a few microseconds, giving a typical dead-time for low-amplitude events of 1.7-1.8 milliseconds. The times listed in Table 3 assume that the longitudinal mode is detected initially. Comparison of the Table 3 values with test data values indicates detected events which should not be included in the data analysis. The following table gives the duration and rise times of a representative set of consecutive high-amplitude channel 7 events $(\geq 57$ $\mathrm{dB}$ ), followed by the relative arrival times of the first four subsequent low-amplitude events $(<57 \mathrm{~dB})$.

Comparison of Table 4 with Table 3 indicates that the first series of Table 4 events, which follow a high-amplitude signal of 1.93 millisecond duration, are all due to internal reflections of a longitudinal wave which triggered the initial event. Events such as that represented by the third example in Table 4 appear to be caused by internally reflected waves travelling with the shear velocity. Other arrival times appear to correspond closely to expected wavemode arrival times, possibly affected also by background noise or interference among modes with similar arrival times, while the number and sequence of reflections which are detected as events are affected by the duration of preceding events combined with the system dead-time.

\subsection{Calibration and Real Acoustic Emission}

Some discussion of the relationship between calibration and real acoustic emission is probably useful. In particular, why should pulser signals be detectable on multiple sensors, while emissions detected during the test were not?

The pulser signals caused by 100 volt and 300 volt excitation were probably primarily Rayleigh and water-borne waves. The water waves propagate through a homogeneous and isotropic medium, and therefore do not suffer losses from interface reflections and diffraction between the injection point and the sensor location. Rayleigh waves in classical plate theory are non-dispersive and contain a singularity in the displacement 
Table 4. Example Relative Arrival Times of Low-Amplitude Channel 7 Events Subsequent to High-Amplitude Events. Class denumeration is to illustrate clustering of relative arrival times of unrelated events.

\begin{tabular}{|c|c|c|c|c|c|c||}
\hline \multirow{2}{*}{$\begin{array}{c}\text { Main Event } \\
\text { Duration/Rise } \\
\text { Time }(\mu \mathrm{sec})\end{array}$} & \multicolumn{5}{|c|}{ Measured Relative Arrival Times (msec) } \\
\cline { 2 - 8 } & Class 1 & Class 2 & Class 3 & Class 4 & Class 5 & Class 6 \\
\hline $1903 / 23$ & 4.8 & - & 6.8 & - & 9.0 & 11.3 \\
\hline $3760 / 7$ & - & - & 6.2 & - & - & 10.2 \\
\hline $3510 / 1301$ & - & 5.7 & - & - & 9.1 & 14.7 \\
\hline $3870 / 21$ & - & - & - & - & 9.5 & - \\
\hline $3850 / 6$ & - & - & - & - & 9.1 & 11.7 \\
\hline $4220 / 711$ & - & - & - & - & - & 12.6 \\
\hline $2750 / 1298$ & 4.9 & - & - & - & - & - \\
\hline $2450 / 8$ & 4.4 & - & - & - & 8.1 & 13.9 \\
\hline $2610 / 1222$ & - & 5.6 & - & - & - & - \\
\hline $4140 / 15$ & - & - & - & - & 9.6 & - \\
\hline $3820 / 24$ & - & - & - & - & - & - \\
\hline $3730 / 14$ & - & - & 6.1 & - & - & - \\
\hline $2590 / 29$ & - & 5.5 & - & 7.5 & - & 10.1 \\
\hline $3350 / 11$ & - & 5.4 & - & - & 8.8 & - \\
\hline
\end{tabular}

amplitude. Since these latter signals travel near and on the surface of the stainless steel cladding, there are few interfaces and structural obstacles to interfere with wave propagation except for the roughness of the surface clad. The wavelength of the Rayleigh acoustic signal is about 5 centimeters or so, hence surface roughness of a much smaller characteristic length would have little effect. However, when a sensor detects a signal from a buried source only the relatively low amplitude longitudinal and shear waves may be detected, since the evanescent longitudinal and shear waves have not been converted to a Rayleigh wave at the surface. Also, a significant amount of signal loss may occur at buried interfaces, transmission losses which would be aggravated by a high angle of incidence. An ability to detect pulser signals at more than one sensor therefore does not imply an ability to similarly detect signals of equivalent strength from buried 
sources, but only indicates that the system is optrating correctly. It follows that detection of events by only one channel during testing is not an indication that the events are caused by noise.

\subsection{Interpretation and Applicability of ASTM Standard E 569 and E 1316}

Appendix A is a copy of relevant pages from section E 569 of the 1991 Annual Book of ASTM Standards [1]. Section 10.1.2 of this publication classifies sources as active and critically active based on event count or emission count, and intense or critically intense depending upon average energy per event, average emission count per event, or average amplitude per event. Terms referring to acoustic emission measurements are defined in part in section E 1316 of the Standards, included as Appendix B of this report.

Emission counts and energy are ambiguous, and usually are indicators of signal amplitude. The acoustic emission counts used to classify activity is a measure of the number of times the oscillating signal crosses the detection threshold during a specific period. Since the output of resonant transducers primarily oscillates at the resonant frequency, the counts obtained from signals with a given shape are co-variant with amplitude (or duration, which is also co-variant with amplitude for this type of signal). An increase in the acoustic emission counts in a given measurement period for invariant-shape, constant amplitude signals can only come about by an increase in the number of events, i.e. the number of individual active sources which produce detectable acoustic emission. Under these latter circumstances, acoustic emission counts and event counts (the number of events detected in a given measurement period) are proportional. The acoustic emission counts therefore may be a measure of the number, the amplitude, or the duration (if the signal shape changes while amplitude and number of events remain constant) of detected events. A significant change in signal shape is unlikely for signals from a given source, therefore a change in shape will usually indicate activation of a second source type, an instrumentation effect (primarily if signals amplitudes are near threshold or saturation), or overlapping events caused by a high emission rate.

Intensity is a measure of average amplitude per event, emission counts per event, or energy per event. As discussed in the preceding paragraph, emission counts per event often indicates the average amplitude or duration. Energy obtained from acoustic emission data is the time integral of squared voltage over the signal duration, usually approximated by the squared amplitude times the duration. For signals of constant shape the energy then becomes proportional to the cubed amplitude, and without initiation of an additional source type, intensity is again a measure of signal amplitude.

Interpretation of the observed data with respect to the ASTM standards is therefore somewhat problematic. An examination of Figures 6 and 9 indicates that all illustrated channels are active, with the probable exception of channel 10. Activity in channels 1 and 4 is discontinuous, but none of the data clearly indicates critical activity as defined 
by the ASTM Standards. Nor, based on amplitude characteristics presented earlier, can active channels be classified as intense or critically intense due to the consistency requirement of section 10.1.2.2 of the Standards, in particular as a single active source is as likely as multiple sources constituting the detected emissions on each active channel.

\subsection{Conclusions}

The presence of acoustic emissions, the probable location of the source of the detected signals within a few feet of the active sensor, and the correlation between acoustic emission behavior and pressurization, prevent crack growth from being eliminated as a possible source process. Channels 1 and 9 may indicate the presence of leakage. An inability to discount crack growth and leakage does not imply that these sources are necessarily present, much less most likely. Innocuous sources unrelated to crack growth may also be responsible for the observed emissions.

The sources of detected acoustic emissions on active channels can be classified as active by ASTM E 569, for which recording of test results for comparison with those of subsequent examination is recommended. Higher classification, for which evaluation by other NDT methods is recommended by the Standards, is not required on the basis of current data.

\subsection{Acknowledgements}

The authors of this report would like to thank ORNL personnel for their outstanding support and cooperation during the course of this program. Oak Ridge managerial and technical personnel continually demonstrated the high degree of capability, knowledge, and efficiency necessary for accomplishment of the program objectives. We wish to acknowledge particularly the help and hospitality provided by Dave Holt, Luther Pugh, Dick Cheverton, Harold Haga, and ORNL radiation training personnel. 


\subsection{References}

1. Annual Book of ASTM Standards, Section 3, Vol. 03.03

2. Hutton, P. H. and J. F. Dawson. 1987. Final Report - Acoustic Emission Monitoring of HFIR Vessel During Hydrostatic Testing, PNL-6345. Pacific Northwest Laboratory, Richland, Washington.

3. Friesel, M. A. 1990. "Acoustic Enission During Intergranular Stress Corrosion Cracking," in Rev. of Prog. in Quantitative NDE, 9B, pp. 1731-1738. 
APPENDIX A: ASTM E 569

A.1 
which is the lowest level measured on th

was measured at $900 \mathrm{psi}$, but due to having achieved higher pressure during an earlier interval, sources such as crack extension which are subject to the Kaiser effect ${ }^{1}$ would be eliminated. Sources subject to the felicity effect ${ }^{2}$ are unlikely to remain active for an extended period at constant pressure, while the observed emission rate at $900 \mathrm{psi}$

${ }^{1}$ Sources subject to the Kaiser effect are not activated until the previous maximum applied stress is exceeded.

${ }^{2}$ The felicity effect describes non-Kaiser effect sources.

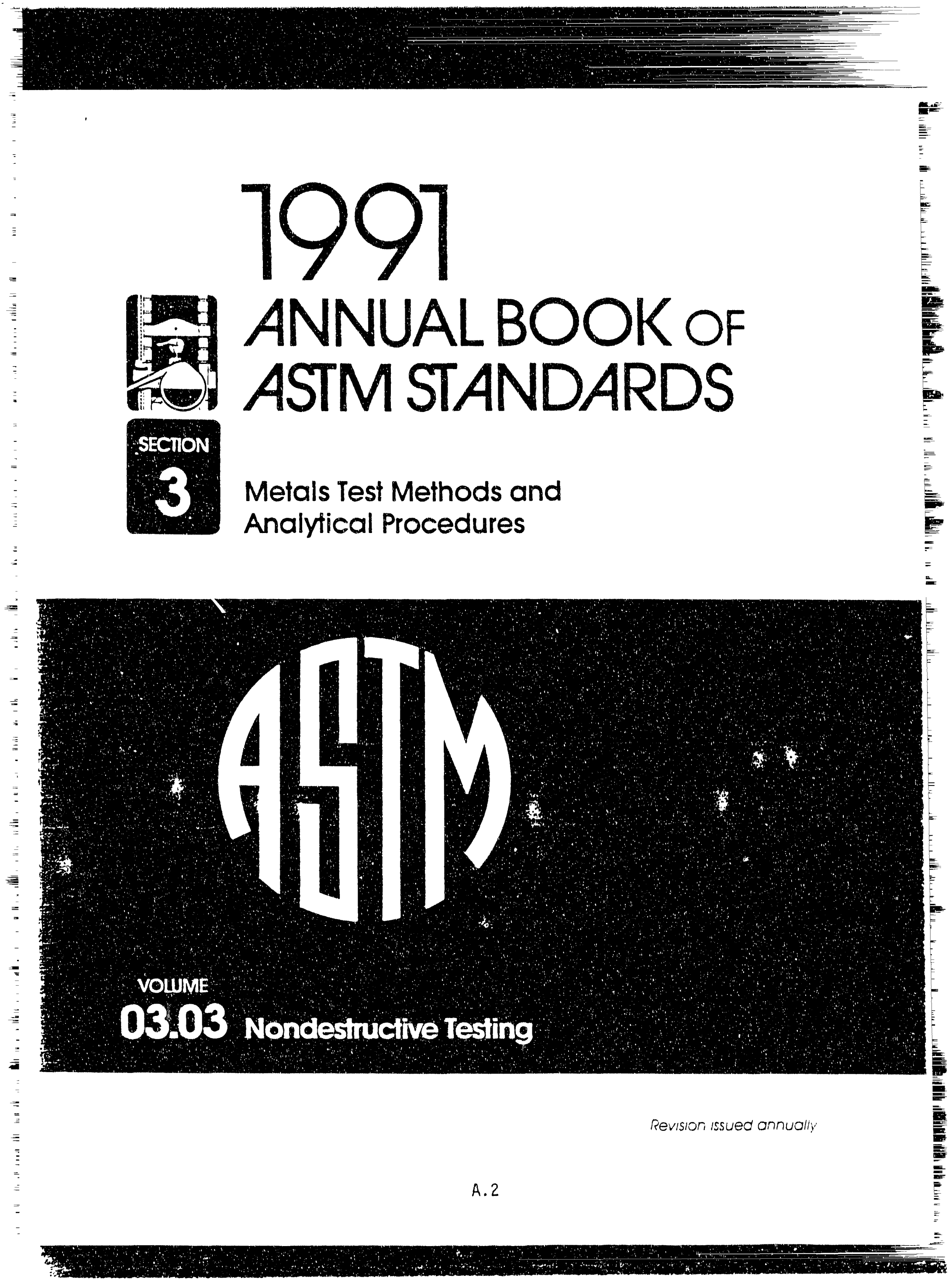




\title{
Standard Practice for Acoustic Emission Monitoring of Structures During Controlled Stimulation ${ }^{1}$
}

\begin{abstract}
This standard is issued under the fixed designation E S69; the number immediately following the designation indicates the year of This standard is issued under the fixed designation E last revision. A number in parenthescs indicates the year of last reapproval. A original adoption or. in the case of revision, the year of last revision. A number in preval.
\end{abstract}

\section{Scope}

1.1 This practice provides guidelines for acoustic emission (AE) examination or monitoring of structures, such as pressure vessels, piping systems, or other structures that can be stressed by mechanical or thermal means.

1.2 The basic functions of an AE monitoring system are to detect. locate, and classify emission sources. Other methods of nondestructive testing (NDT) may be used to further evaluate the significance of acoustic emission sources.

1.3 This standard may involve hazardous materials, operations, and equipment. This standard does not purport 10 adaress all of the safety problems associated with its use. It is the responsibility of whoever uses this standard to consult and establish appropriate safety and health practices and deter. mine the applicability of regulatory limitations prior to use.

\section{Referenced Documents}

2.1 AST.M Standards:

E 610 Terminology Relating to Acoustic Emission?

E 650 Guide for Mounting Piezoelectric Acoustic Emission Sensors ${ }^{2}$

E 750 Practice for Measuring the Operating Characteristics of Acoustic Emission Instrumentation"

\section{Summary of Practice}

3.1 Acoustic emission examination of a structure usually requires application of a mechanical or thermal stimulus. Such stimulation produces changes in the stresses in the structure. During stimulation of a structure, AE from discontinuities (such as cracks and inclusions) and from other areas of stress concentration, or from other acoustic sources (such as leaks, loose parts, and structural motion) can be detected by an instrumentation system, using sensors which. when simulated by stress waves. generate electrical signals. The sensors are acoustically coupled to the suriace of the structure, usually by means of a couplant material that improves the transmission of stress waves to the sensor. The detected AE signals can be electronically conditioned and processed. to facilitate recording or visual display of the amplitude and location of the emission sources. increasing the internal pressure of a vessel is a common stimulus.

\footnotetext{
This practice is under the junsdiction of ASTM Committee E.? on Vnnde. strucuve Testing and, the dired responsibility of Subcommitiet E07.04 on acousic Emission.

Cument edition approved Oct. 25. 1985. Published December 14\$5. Oneunally puhishect as E 569 - :0. Last previous edition E $569-82$.

$\because$ innual Bersk of tSTM Siandards. Vol 03.03
}

Application of localized forces to the exterior boundary of piping and similar structural components is an example of another possible stimulus.

3.2 In addition to immediate evaluation of the emissions detected during the application of the stimulus, a permanent record of the number and location of emitting sources and the relative amount of $A E$ detected from each source provides a basis for comparison with sources detected during the test and during subsequent stimulation.

\section{Order Information}

4.1 If AE monitoring in accordance with this practice is performed as a service, the following items are subject to agreement between the purchaser and supplier:

4.1.1 The Area(s) 10 Be Monitored-Many applications will require an arrangement of sensors such that all areas of the structure are monitored. In other applications, only a portion of the structure may reyuire monitoring.

4.1.2 The stimulation schedule and other stimulation parameters.

4.1.3 The person(s) responsible for stopping or holding the test if unexpected or unusual AE activity response patterns are detected.

4.1.4 The date and location of the intended examination and the expected ambient conditions.

4.1.5 The criteria to be used for interpreting, classifying, and evaluating $A E$ indications and response patterns, if different from that described in this practice.

4.1.6 Disposition of records or other test data and contents of the test report.

4.1.7 Survey of the structure for extraneous background noise that could preclude effective testing.

\section{Personnel Qualification}

\$.1 The acoustic emission examination shall be performed by qualified personnel. Qualification should be based on demonstrated skill, training, and experience.

\section{Examination Preparation}

6.l Before the examination begirs. make the following preparations for AE monitoring:

6.1 .1 Determine the type. number. and placement 0 sensors. This requires inowledge of both matenal ar. physical characteristics of the structure and the features the instrumentation. This determination is also depender. upon the required precision and accuracy of the examina tion.

6.1.2 Establist communications between the contr point for the application of the stimulus and the $A E$ : control center. 
6.1.3 Provide a means for continuously recording a meare of the stimulus.

6.1.4 Identify potential sources of extraneous acoustic ise. such as vibration. friction, and nuid nou: Such urces may require acoustic isolation or control. in order it to mask valid acoustic emissions.

6.1.5 Altach the sensors: both the couplant and scrising rice must be compatible with the surface conditions and le composition of the structural material being examined ee Guide E 6.50).

6.1.6 Calibrate the AE monitoring system in accordance ith Section 7. The calibration shall be performed as a ininimum, before and after the test. Additional calibration nay be performed during the test at the discretion of the wner or his designee.

\section{- Calibration of A pparatus}

7.1 Calibrate the system used for AE monitoring and data nterpretation in accordance with a written procedure (see ractice $E$ 750).

7.2 Background noise is normally minimized by acoustially isolatung the structure to an extent that is compatible with the chosen stimulation procedure. After all sensors are connected and operational. the AE monitoring system can then be calibrated at test time using an AE simulator. This simulator should be capable of repeatedly producing a iransient elastic wave having an amplitude representative of the $A E$ to be detected. Using the $A E$ simulator. determine both the sensitivity and locational accuracy of the $A E$ monitoring system.

7.2.1 Sensitivity Calibration-In order to set the sensivivity for a sensor channel. locate the acoustic emission simulator at a selected distance from the sensor. Monitor the channel's response to the simulated emission. and adjust the channel gain or threshold to obtain a specified response. Then repeat this procedure for each AE sensor on the siructure. in cases where this approach is economically unattractive or technically infeasible. the above procedure may be performed on an alternative structure (comparison block). using the same cable required for attachment to the test structure. A second alternative method, that may be applied after the sensors are mounted on the structure. is to apply an electrical pulse to each sensor in tum. and from analysis of the response of the other sensors. make any' adjustments necessany to bring all channels to the same sensitivity. If the sensitivity of individual channels must be altered due to background noise or other reason, each change in sensitivity shall be recorded.

7.2.2 Location Calibration-Check the operation of the $A E$ source location function by analyzing simulated $A E$ signals from several locations on the structure. as well as from any specific critical locations. For each placement of the simularor, determine and record the precision and accuracy of the AE location function. It should be noted that the accuracy of locating the simulated source will not necessarily be the same as for locating a real $A E$ source.

\section{Examination Procedure}

8.1 Acoustic emission data may be accumulated during or after stimulation of the structure. or both.
8.1.1 During application of the stimulus. the locations of acoustic sources are usually determined through analysis of the times of arrival of AE signals at multiple sensors. Such analysis may he performed through the use of an analog or digital computer. The computer accurnulates and analyzes data over a specified parametric range. Examples of parameters are pressure, time, and stress. As the stimulus is applied. record the number and location of emitting sources and the amount of $A E$ detected from each source. The $A E$ rate at one or more sensors ma! be monitored and displayed in real time during stimulation. If the acoustic emission activity. intensity. or a similar AE quantiny show's an increase such as 10 cause concern. notify the ou'ner of the structure or his designee immediately. It shall be the owner's decision to reduce. hold. or stop the test. The cause of the AE increase shall be investigated before continuing the stimulation schedule.

8.1.2 Continuous emission from any leak in a structure stimulated by pressure can mask acoustic emission from sources near the leak. Efrects of leaks on acoustic emission measurements should be eliminated to adequately test pressure boundaries. Knowledge of attenuation in the structure and the response of sensors affected by leak noise may help localize the leak.

8.1.3 Following the test, repeat the calibration in accordance with Section 7. Record any changes in the resulting gain settings or in the source location performance. relative to the previous calibration, and make appropriate compensation, if feasible, during data interpretation.

\section{Examination Records}

9.1 All calibration data and instrument adjustments. including equipment description and performance data. shall be included in the records of the examination and be signed by the responsible party. The information recorded should be sufficient to permit complete reanalysis of the resulis. This information should include. but not be limited to:

9.1.1 Material and physical characteristics of the struc. ture.

9.1.2 Sensor specifications. including size. sensitivity. fre. quency response. method of attachment, type of couplani. type and length of connecting cables.

9.1.3 Sensor locations.

9.1.4 Functional descriptions of signal conditioners. proc. essors. and read-out equipment.

9.1.5 Schedule, procedures. and results of all calibrations.

9.1.6 Method of stimulation and examination schedule.

9.1.7 Permanent data record of the measured AE signal parameters, in analog or digital form.

\section{Interpretation of Results}

10.1 All results shall be summarized on an appropriate layoui map, displayed or tabulated or both. for ready reference and interpretation. This layout or tabulation shall display the location and classification of each source with pertinent comments.

10.1.1 Sourcc Location-All location data resulting from analysis shall be presented in a manner consistent $u$ ith the previously established calibration accuracy.

10.1.2 Source Classification-Sources are usually classi. fied with respect to their acoustic acrivity and intensing.

\subsubsection{1}

by event $\mathrm{cr}$ be active if increase wil considered event coun consistently rate of cha respect 10 constant st

10.1.2.2

average del per event. considered measure $c$. average int can be calc An intense intensity $c$ with time that. if the of the sol therefore : this case. compariso

10.1.2.3 character: consideres 


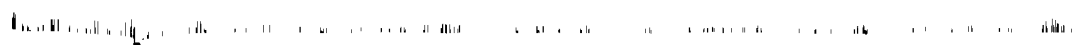

ns of

sis of

Such

og or

dlyzes

ram-

plied,

$d$ the

it one

btime

n:en-

as to

ir his

on to

irease

iation

icture

from

ission

pres-

Icture

$\because$ help

cordg gain to the ion, if

is. in. . shall igned hould ssults. ):

struc.

$\checkmark$ ire. plant,

proc-

ltions.

sule.

signal

priale

ready

r shail

$\therefore$ with

$\therefore$ trom

in the

classi...
10.1.2.1 A source's acoustic activity is normally measured by event count or emission count. A source is considered to be active if its event count or emission count continues to increase with increasing or constant stimulus. A source is considered to be critically active if the rate of change of its event count, or emission count, with respect to the stimulus. consistently increases with increasing stimulation, or if the rate of change of its event count, or emission count, with respect to time, consistently increases with time under constant stimulus (see Fig. 1).

10.1.2.2 Preferred intensity measures of a source are its: average detected energy per event, average emission count per event. or average amplitude per event. A source is considered to be intense if it is active and its intensity measure consistently exceeds, by a specified amount, the average intensity of active sources. The intensity of a source can be calculated for increments of the stimulus or of events. An intense source is considered to be critically intense if its intensity consistently increases with increasing stimulus, or with time under constant stimulus (see Fig. 2). It is noted that, if there is only one active source, the intensity measure of the source is the average intensity of all sources, and therefore the intrinsic comparison no longer is applicable. In this case, it is necessary to classify the source through comparison with results from similar tests.

10.1.2.3 In addition to activity and intensity, another characteristic of each detected AE source that shouid be considered for source classification is the size of the "region" of the located source. The clustering of the located events from a sharp discontinuity. such as a crack, is usually dense. while regions of plastic deformation associated with, for example. corrosion pits, result in source areas that show more uncertainty in the definition of their size, the events being contained rather sparsely in the region. In most cases, a growing.crack is considered to be the more serious defect. However, activity and intensity may not suffice for distinguishing between the two. Normally, there is subjective judgment on what size of location bundle or clusier constitutes an isolated source.

10.1.3 Source Evaluation-Sources are usually evaluated by their activity or intensity.

10.1.3.1 Sources considered to be critically active. critically intense. or both are indicative of questionable structural integrity and, if possible, shall be evaluated by other NDT methods.

10.1.3.2 Sources considered to be intense are indicative oi possible flaw growth and. if possible. should be evaluated by other NDT methods.

10.1.3.3 Sources considered to be active but not intense shall be recorded for comparison with sources detected during subsequent examinations.

10.1.3.4 Sources considered to be of low activity and intensity are not usually required to be further evaluated or subsequently correlated.

\section{Report}

11.1 A report should at least contain the examination record and the interpretation of results.

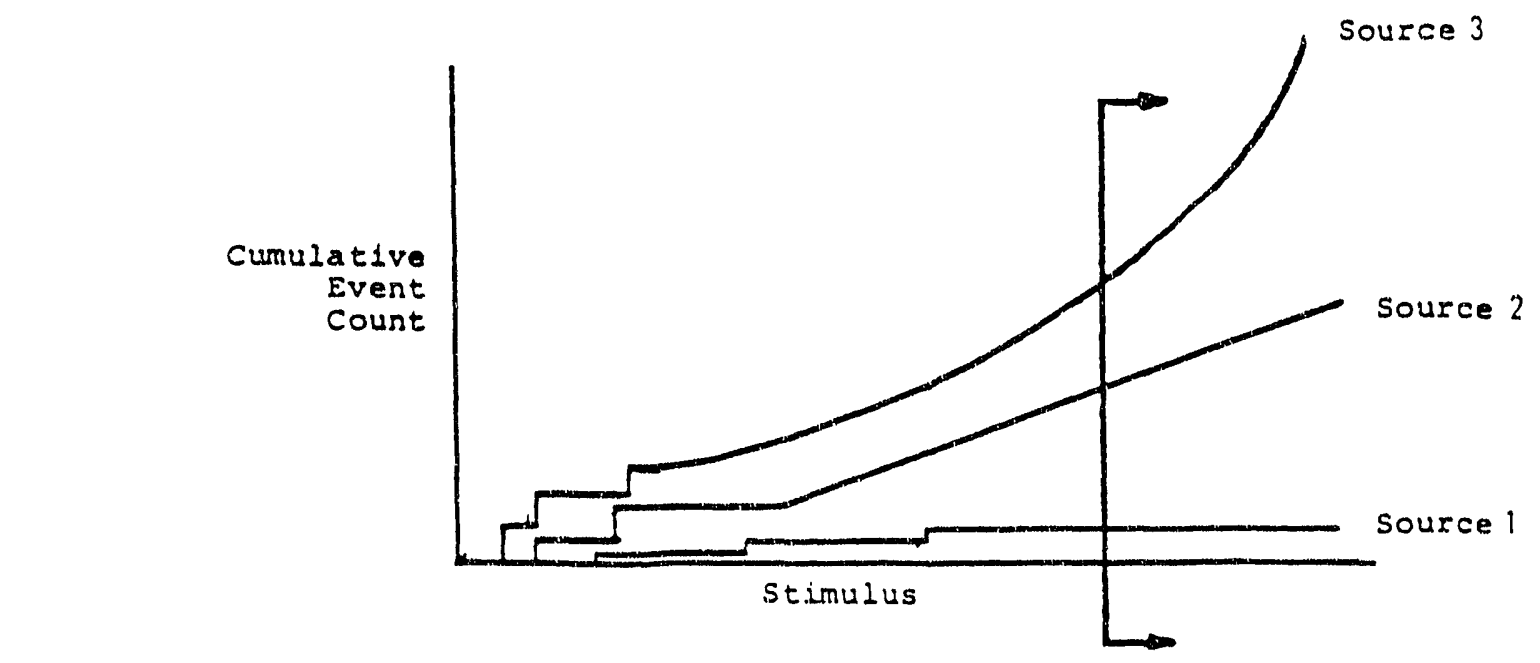

Nore- To ine nght of the venical line. Source $i$ is inactive. Source 2 is active, and Source 3 is cntically active

FIG. 1 Schematic Representation of Three Different Source Types 


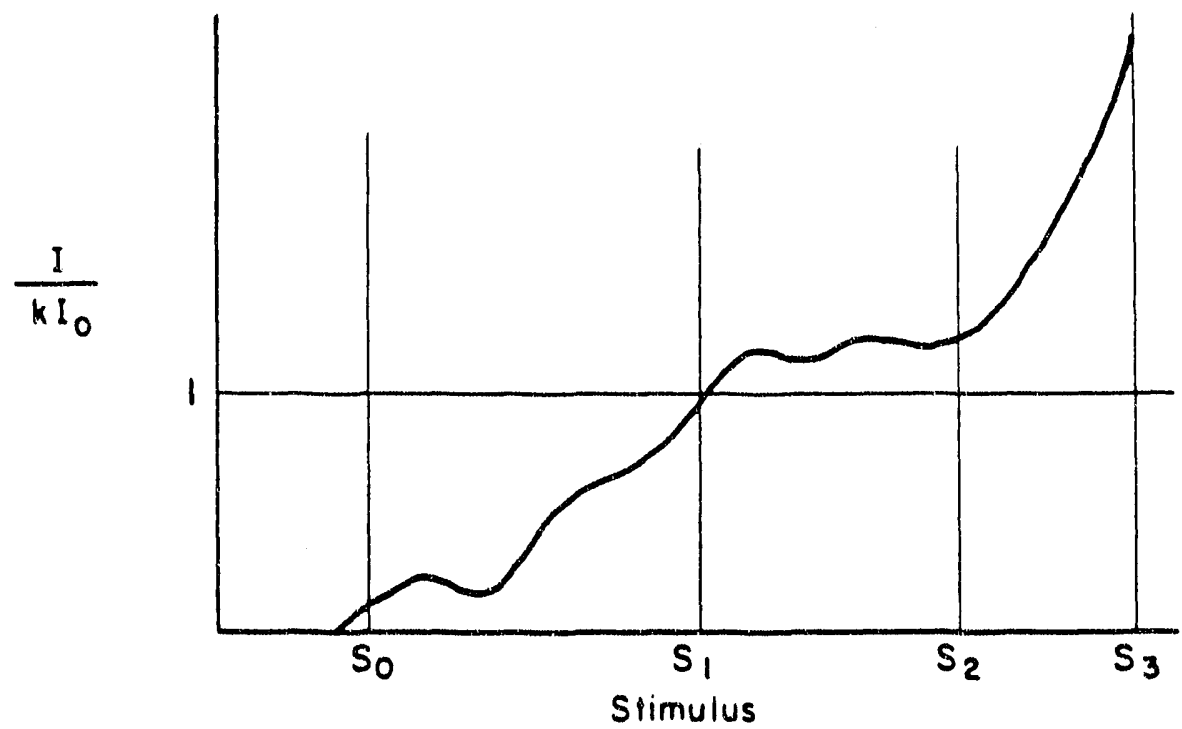

NOTE-Four different regions are shown: prior $10 S_{0}$, the source was hactive; between $S_{0}$ and $S_{1}$, the source was of low intensity; between $S_{1}$ and $S_{2}$, the source is dassified as intense; between $S_{2}$ and $S_{3}$, the source is classified as critically intense.

FIG. 2 Source Intensity, l, Divided by a Weighted Average Intensity of All Sources, klo, Plotted Against the Stimulus, S

Thu Anerican Society for Tasting and Materials takes no postion respecting the valiulty of any patont rights asserted in connection wh any hem mentioned in this standard. Users of ihis standard are expressly advised that determination of the validity of any such patemt righis, and the risk of infringement of such rights, are entirely their own responsibitity.

This standerd is bubject to revision a any time by the responslble technical commitree and must be reviowed overy fivo years and If not revised, ather reepprovad or withdrawn. Your comments are invited either for rovision of this standard or for additional standards and should be oodressed to ASTM Heedquerters. Your comments will receive careful consideration at a meoting of the responsibte technical committee, which you may attend. If you teel that your comments have not received a fair hoaring you should make your viows known to the ASTM Commitree on Standards, 1916 Aace ST., Philadolphia, PA 19103. 
APPENDIX B: ASTM E 1316

B.1 


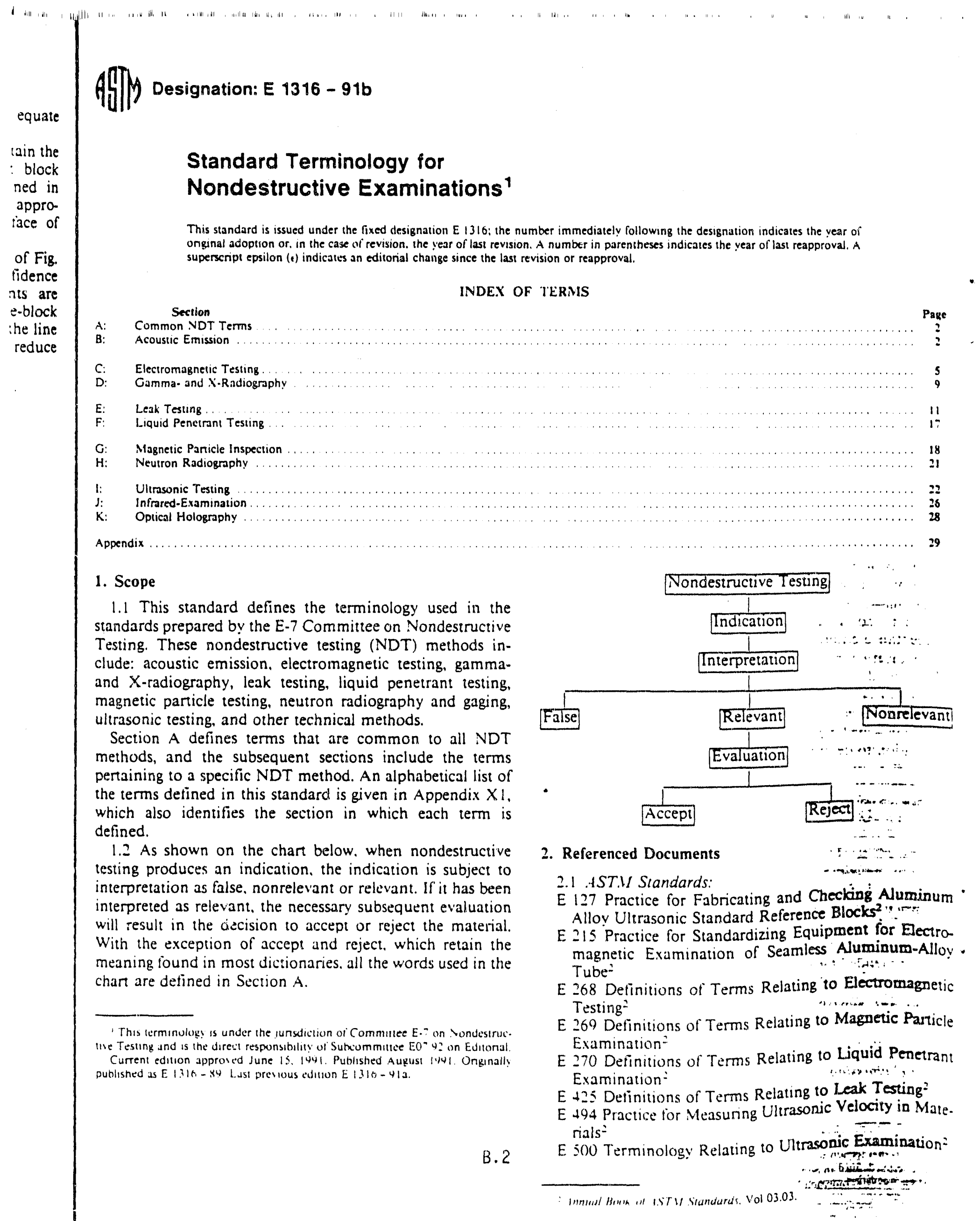


E 545 Method for Determining Image Quality in Direct Thermal Neutron Radiographic Testing"

E so6 Practice for Electromagnetic (Eddy-Curtent) Sorting of Ferrous Metals 2

E 586 Terminology Relating in Industrial Radiolng:

E 610 Definitions of Terms Relating to Acoustic Enis$\operatorname{sion}^{2}$

E 664 Practice for Measurement of the Apparent Attenuation of Longitudinal Ulirasonic Waves by Immersion Method:

E 748 Practices for Thermal Neutron Radiography of Materials:

E 750 Practice for Characterizing Acoustic Emission Instrumentation ${ }^{2}$

E 804 Practice for Calibration of the Ultrasonic Test System by Extrapolation Between Flat-Bottom Hole Sizes?

E 1033 Practice for Electromagnetic (Edd)-Current) $E_{\lambda \text { - }}$ amination of Type F- Continuously Welded $(\mathrm{CW}$ ) Ferromagnetic Pine and Tubing Above the Curie Temperature?

E 1067 Practice for Acoustic Emission Examination of Fiberlass Reinforced Plastic Resin (FRP) Tanks/ 'essels:

E 1118 Practice for Acoustic Emission Examination of Reinforced Thermosetting Resin Pipe (RTRP):

E 1149 Terminology Relating to NDT hy Infrared Thermography:

E 1213 Test Method for Minimum Resolvable Temperiture Difference for Thermal Imaging Sy'stems

\section{Significance and Use}

3.1 The terms found in this proposed standard are intended to be used uniformly and consistently in all nonde. structive testing standards. The purpose of this standard is 10 promote a clear understanding and interpretation of the NDT standards in which they are used.

\section{Section A: Common NDT Terms ${ }^{3}$}

defect-a discontinuity or group of discontinuities whose indications do not meet specified acceptance criteria.

discontinuity-an intersuption, which may be either intentional or unintentional in the physical structure or configuration of a par.

evaluation-a review, following interpretation of the indications noted. to determine whether they meet specified acceptance criteria.

false indication, $n$-an N.DT indication that is interpreted to be caused by a discontinuity at a location where no discontinuity exists.

flan:-see defect.

indication-evidence of a discontinuity that requires interpretation to determine its significance.

The terms defined in Section A are the direct responsibility of Subcommittec E07.92. Editonal Revien.

\section{Section B: Acoustic Emission (E 610, E 750, E 1067, and E 1118) 4.5}

acoustic emission (AE) - the class of phenomena whereby transient elastic waves are generated by the rapid release of energy from localized sources within a material. or the transient waves so generased. Acoustic emission is the recommended term for general use. Other terms that have been used in AE literature include (1) stress wave emission, (2) microseismic activity, and (3) emission or acoustic emission with other qualifying modifiers.

acoustic emission channel-see channel, acoustic emission.

acoustic emission coum (emission count) ( $\mathrm{N})$-see count, acoustic emission.

\footnotetext{
- The boidiace designations in parentheses indicate the standards from which un lerms in that secuon were deried.

The terms defined in Section B are the direct responsibilu! of Subcommulue
} EO?.11 on Acoustic Emission Method interpretation-the determination of whether indications are relevant or nonrelevant.

Nondestructive Testing (NDT), $n$-the development and application of technical methods to examine materials or components in ways that do not impair future usefulness and serviceability in order to detect, locate, measure and evaluate discontinuities, defects and other imperfections; to assess integrity, properties and composition: and to measure geometrical characters.

Nondestructive Evaluation-see Nondestructive Testing

Nondestructive Examination-see Nondestructive Tesling.

Nondestructive Inspection-see $N^{\prime}$ ondesiructive Testing.

nonrelevant indication, $n$-an NDT indication that is caused by a condition or type of discontinuity that is not rejectable. False indications are non-relevani.

relevant indication, $n$-an NDT indication that is caused by a condition or tspe of discontinuity that requires evalua. ijon. acoustic emission count rale-see count rate. acoustic emission (emission rate or count rate) $(\mathrm{N})$.

acoustic emission event-see event, acoustic emission.

acoustic emission event energl- - see energy, acoustic event. acoustic emission sensor-see sensor, acoustic emission.

acoustic cmission signal ampiitude-see signal amplitude, acoustic emission.

acoustic emission signal (emission signal)-see signal. acoustic emission.

acoustic emission signature (signature)-see signature. acoustic emission.

acoustic cmission transducer-see sensor, acoustic emission. acoustic emission raveguide-see waveguide, acoustic emission.

AE signal duration-the time between AE signal start and AE signal end.

$A E$ signal end - the recognized termination of an AE signal. usually defined as the last crossing of the threshold by that 


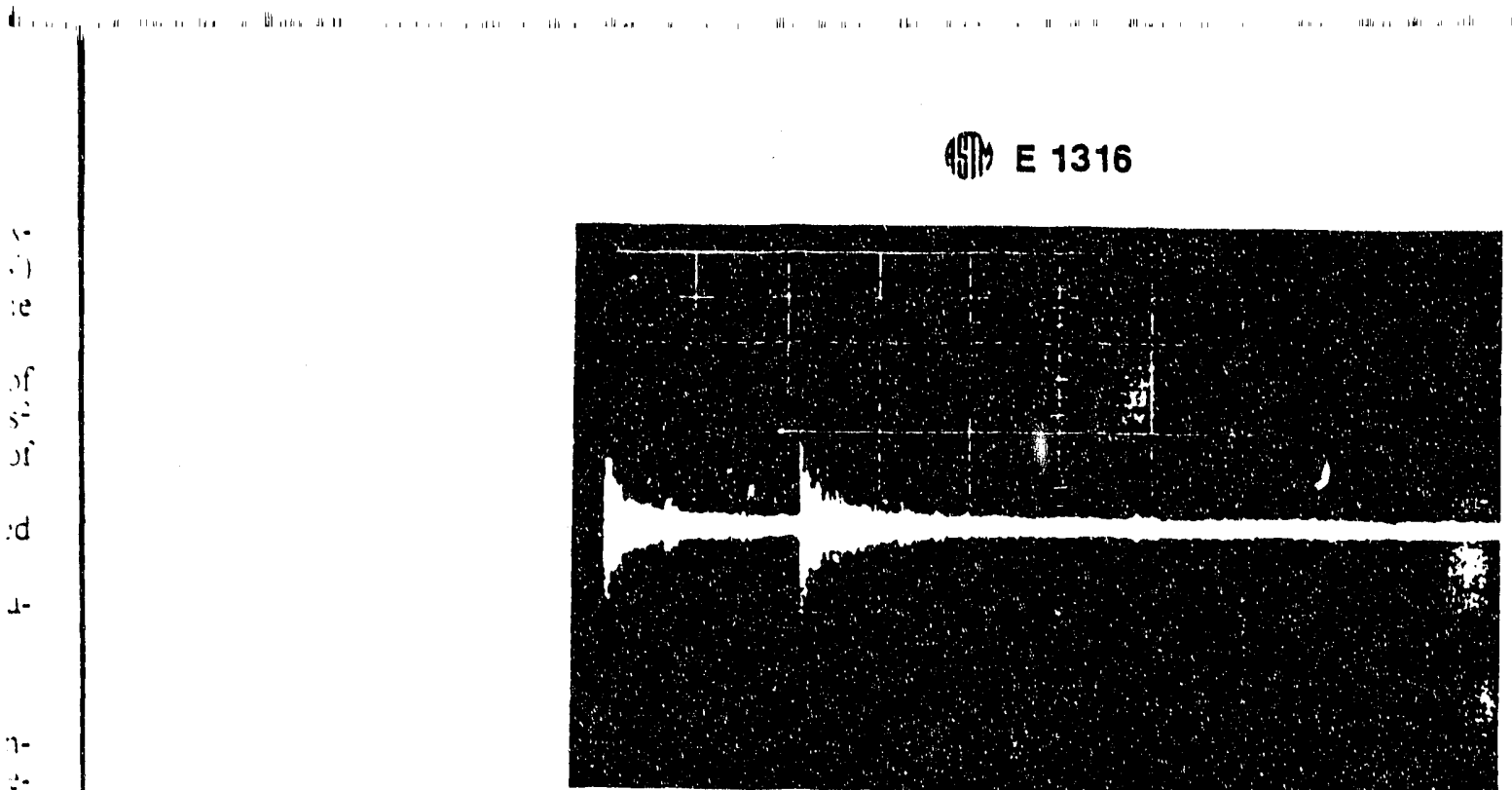

FIG. 1 Burst Emission on a Continuous Emission Background. (Sweep Rate $-5 \mathrm{~ms} / \mathrm{cm}$.)

signal.

AE signal generator - a device which can repeatedly induce a specified transient signal into an AE instrument.

$A E$ signal rise time-the time between AE signal start and the peak amplitude of that AE signal.

AE signal start-the beginning of an AE signal as recognized by the system processor, usually defined by an amplitude excursion exceeding threshold.

arrival time interval $\left(\Delta l_{i j}\right)$-see interval, arrival time.

burst emission-see emission, burst.

channel, acoustic emission-an assembly of a sensor, preamplifier or impedance matching iransformer, filters secondary amplifier or other instrumentation as needed, connecting cables, and dedector or processor.

Note I-A channel for examining liberglass reinforced plastic (FRP) may unlize more than one sensor with associated electronics. Channels may be processed independently or in predetermined groups having similar sensitivity and frequency characteristics.

continuous emission-see emission, continuous.

count, acoustic emission (emission count) $(N)$-the number of times the acoustic emission signal exceeds a preset threshold during any selected portion of a test.

count, event $\left(\mathbf{N}_{e}\right)$ - the number obtained by counting each discerned acoustic emission event once.

count rate, acoustic emission (emission rate or count rate) $(N)$ - the time rate at which emission counts occur.

count, ring-down-see count, acoustic emission, the preferred term.

couplant - a material used at the structure-to-sensor interface to improve the transmission of acoustic energy across the interface during acoustic emission monitoring.

cumulative iacoustic emission) ampliunde distribution F(I)-see distribution, amplitude, cumulative.

cumulative lacoustic emission) threshold crossing distribution $F_{1}(l)$-see distribution, threshold crossing, cumulative.

dead time-any interval during data acquisition when the instrument or system is unable to accept new data for any reason. $(\text { E } 750)^{n}$

\footnotetext{
"The designaltons in parentheses following the terms indliate the iSTV
} idndards linem which the terms were denved. differential (acoustic emission) amplitude distribution $F(I)$ see distribution, differential (acoustic emission) amplitude $\mathbf{f}(\mathbf{V})$.

differential (acoustic emission) threshold crossing distribution $f_{t}\left(V^{\prime}\right)$-see distribution, differential (acoustic emission) threshold crossing.

distribution, amplitude, cumulative (acoustic emission) $F(V)$ - the number of acoustic emission events with signals that exceed an arbitrary amplitude as a function of amplitude $\mathrm{V}$.

distribution, threshold crossing, cumulative (acoustic emission) $F_{i}(V)$ - the number of times the acous. is emission signal exceeds an arbitrary threshold as a function of the threshold voltage $(V)$.

distribution, differential (acoustic emission) amplitude $f(V)$ - the number of acoustic emission events with signal amplitudes between amplitudes of $V$ and $V+\Delta V$ as a function of the amplitude $V, f(V)$ is the absolute value of the derivative of the cumulative amplitude distribution $F(V)$.

distribution, differential (acoustic emission) threshold crossing $f_{f}(V)$ - the number of times the acoustic emission signal waveform has a peak between thresholds $V$ and $V+$ $\Delta V$ as a function of the threshold $V . f_{t}(V)$ is the absolute value of the derivative of the cumularive threshold crossing distribution $F_{1}(V)$.

distribution, logarithmic (acoustic emission) amplitude $g(V)$ - the number of acoustic emission events with signal amplitudes between $V^{\prime}$ and $\alpha V^{\prime}$ (where $\alpha$ is a consiant multiplier) as a function of the amplitude. This is a variant of the differential amplitude distribution. appropriate for logarithmically windowed data.

dynamic range-the difference. in decibels. between the overload level and the minimum signal level (usually fixed by one or more of the noise levels. low-level distortion. interference, or resolution levell in a svstem or sensor.

emission. burst-a qualitative description of the discrete signal related to an individual emission event occurring within the material.

Vort :- Lse of the term hurst emission is recommended onle tir describing the qualitalive appedarance of emisson ignals. Figure shou's an oscilloscone trace of hurst :mission signals on a hachernund of continumus amission. 


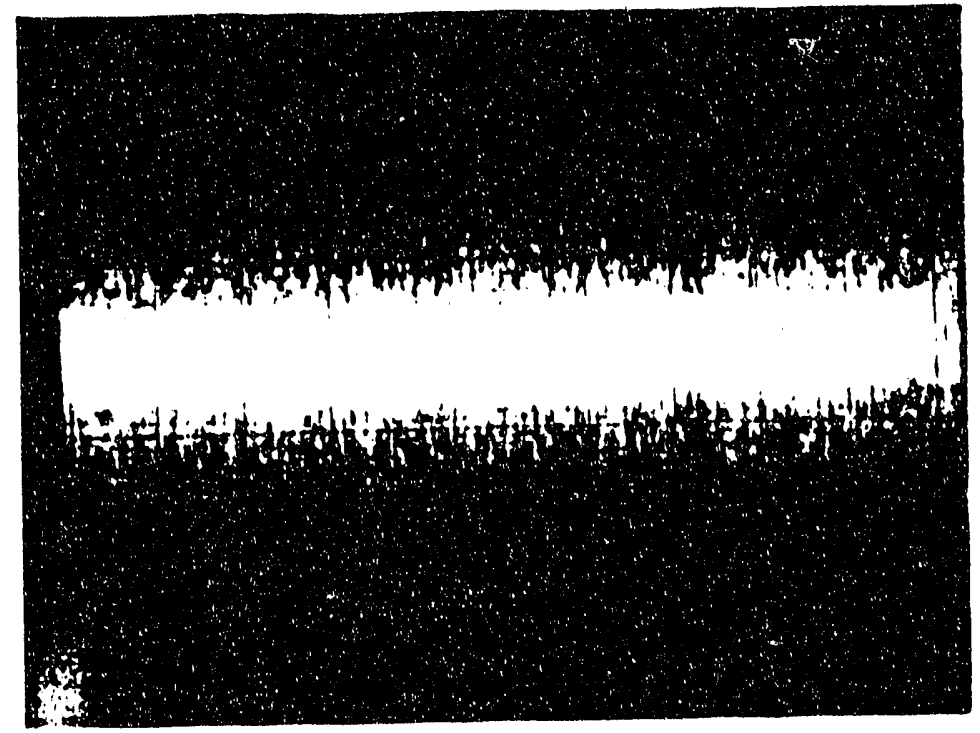

instr.

noati

lis:

75

hit-

s!:

inter

de

an

Kais

a

les

$\log a 1$

$g^{\prime \prime}$;

pli

overl

FIG. 2 Continuous Emirsion. (Sweep Rate $-5 \mathrm{~ms} / \mathrm{cm}$.)

or

exi

pres:

res

pri

proci

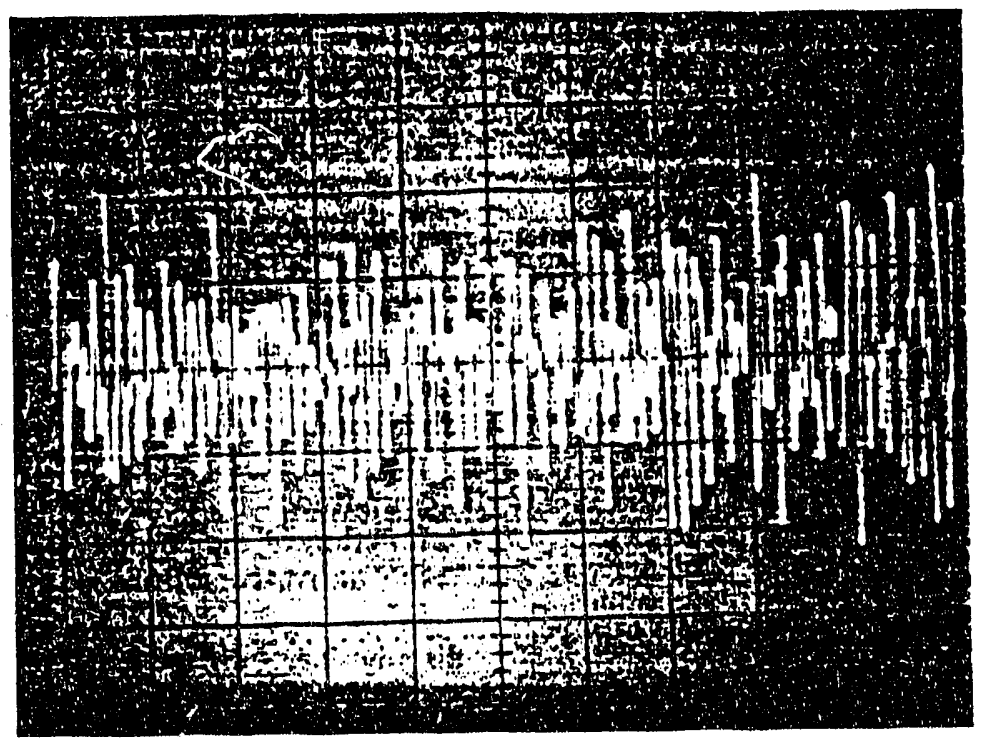

FIG. 3 Continuous Emission. (Sweep Rate $-0.1 \mathrm{~ms} / \mathrm{cm}$.)

emission, continuous - a qualitative description of the suszained signal level produced by rapidly occurring acoustic emission events.

NOTE 3- Use of the term comimuous emission is recommended only for describing the qualitative appearance of emission signals. Figures 2 and 3 show oscilloscope traces of continuous emission signals at two different sweep rates.

energy, acoustic emission event-the total elastic energy released by an emission event.

evaluation threshold-a threshold value used for analysis of the examination data. Data may be recorded with a sy'stem examination threshold lower than the evaluation inreshold. For analysis purposes, dependence of measured data on the sisiem examination threshold must be taten into consideration.

event, acoustic emission (emission event)-a local material change giving rise to acoustic emission. event count $\left(\Lambda^{\circ} \mathrm{c}\right)$--see count, event.

event count rate ( $\mathrm{Nic}_{\mathrm{C}}$-see rate, event count.

examination area-that porion of a structure being monitored with acoustic emission.

examination region-that portion of the test article evaluated using acoustic emission technology.

Felicity effect-the presence of acoustic emission. detectable at a fixed predetermined sensitivity level at stress levels below those previously applied. (E 1067)

Felicity effect-the presence of detectable acoustic emission at a fixed predetermined sensitivity level at stress levels below those previously applied.

Felicity ratio-the ratio of the stress at which the Felicity effect occurs to the previously applied maximum stress. (E 1067, E 118 )

Note 4-The fixed sensitivity level will usually be the same as was used for the previous loading or test. (E 1118 ) 
instrumentation dead time-see dead time, instrumentation. floating threshold-any threshold with amplitude established by a time average measure of the input signal. ( $E$ 750)

hit-any signal that exceeds the threshold and causes a system channel to accumulate data. (E 750)

interval, arrival time $\left(\Delta t_{i j}\right)$-the time interval between the detected arrivals of an acoustic emission wave at the $i t h$ and $j$ th sensors of a sensor array.

Kaiser effect - the absence of detertable acoustic emission at a fixed sensitivity level, until previously applied stress levels are exceeded.

logarithmic (acoustic emission) amplitude distribution $g(l)$-see distribution, logarithmic (acoustic emission) amplitude.

overload recovery time-an interval of nonlinear operation of an instrument caused by a signal with amplitude in excess of the instrument's linear operating range.

pressure, design-pressure used in design to determine the required minimum thickness and minimum mechanical properties.

processing capacity - the number of hits that can be processed at the processing speed before the system must interrupt data collection to clear buffers or otherwise prepare for accepting additional data.

processing speed-the sustained rate (hits/s), as a function of AE signals can be continuously processed by a system without interruption for data transport.

rate, event count $(\dot{\mathrm{Ne}})$ - the time rate of the event count.

rearm delay' 'ime-see time, rearm delay.

ring-down count-see count, acoustic emission, the preferred term.

sensor, acoustic emission-a detection device, generally piezoelectric, that transforms the particle motion produced by an elastic wave into an electrical signal.

\section{Section C: Electromagnetic Testing}

absolute coil-a coil (or coils) that respond(s) to the total detected electric or magnetic properties, or both of one section of a part undergoing electromagnetic test without comparison to another section of the part, or to another part. (E 268)

absolute coil-a coil (or coils) that respond(s) to the total detected electric or magnetic properies, or both. of a part or section of the test part without comparison to another section of the part or to another part. (E 506)

absolute measurements - in electromagnetic testing, measurements made withoug a direct reference using an absolute coil in contrasi to differential and comparative measurements. (See also absolute crill).

absolute readout-in electromagnetic testing. the signal outpu: of an absolute coil. (See also absolute coil).

absolute system-an electromagnetic testing system that uses a coil assembly and associated electronics to measure the total electromagnetic properties of a test part without

"The terma actined in Section $C$ are the dired responsibility of Sutorommitte the parameter set and number of active channels, at which

signal, acoustic emission (emission signal)-an electrical signal obtained by detection of one or:more acoustic emission events.

..

signal amplitude, acoustic emission-the peak voltage of the largest excursion attained by the signal waveform from an emission event.

signal overload level-that level above: which : operation ceases to be satisfactory as a result of signal distortion. overheating, or damage.

74.

signal overload point - the maximum inpur signal amplitude at which the ratio of output to input is observed to remain within a prescribed linear operating range tiena:-2: :

signature, acoustic emission (signature) $\ddot{-a}$ charocteristic set of reproducible attributes of acoustic emission signals associated with a specific test article as observed with a particular instrumentation system under specified test conditions.

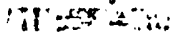

stimulation-the application of a stimulus such is force pressure, heat, etc., to a test article to cause activation of acoustic emission sources.

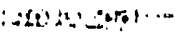

system examination threshold-the electronic instrument thresholid (see evaluation threshold) which data will be detected.

transducers, acoustic emission-see sensor, isconstic emission.

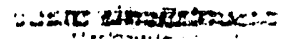

voltage threshold - a voltage level on an electronic comparator such that signals with amplitudes lare thas this level will be recognized. The voltage threshold may be user adjustable, fixed, or automatic floating (E-750) 2 tya:

waveguide, acoustic emission-a device that coupies elastic energy from a structure or other test objoch to a remotely mounted sensor during AE monitoring An example of an acoustic emission waveguide would be a solid wire of rod that is coupled at one end to a monitored structare, and to a sensor at the other end.

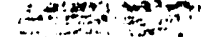

- mstrot 5

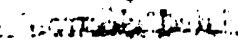

(E 215, E 243, E 268, E 366, E 1033) ${ }^{7}$ direct comparison to another section of the part or to

(E 215, E 243, E 268, E 366, E 1033) ${ }^{7}$ direct comparison to another section of the part or to direct comparison to another section or the
another part. (See also absolute coil.)

absolute system-a system that uses a coil asembiy and associated electronics to measure the total dectromagnetic properties of a test part without direct comparison to properties of a test part with a another part (see sabsolute coil). (E 566)

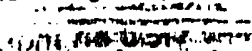

acceptance level-a test level above or below which test specimens are acceptable in contrast to rejection level.

acceptance limits - test levels used in electromagnetic inspec. tion that establish the group into which a material under est belongs.

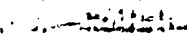

acceptance limirs - iest levels used in electromagnetic sorting which establish the group into which the material uncier lest beliungs. ( $E \$ 00$ )

noistirsti

cceptance standard-in tubing inspection, a tube used 10 establish the acceptance level with artificial discontinuities as specified in the applicable product standard.acceptance standard-a tube with artificial discontinuities specified in the applicable product standard used to estahlish the acceptance level. (E 215) E0) 07 an Electromannelic betherds.

\section{B. 6}




\section{DISTRIBUTION}

No. of

Copies

\section{OFFSITE}

12 DOE/Office of Scientific and

Technical Information

5 D. C. Holt

Halliburton NUS Env. Corp.

800 Oak Ridge Turnpike

Jackson Plaza C-200

Oak Ridge, TN 37830

\section{QNSITE}

DOE Richland Field Office

\section{J. J. Sutey, A5-90}

16 Pacific Northwest Laboratory

D. M. Boyd, K5-10

J. F. Dawson, K2-31

S. R. Doctor, K2-31

M. A. Friesel, K2-31 (5)

B. P. Hildebrand, K2-31

P. H. Hutton, K2-31

Publishing Coordination

Technical Report Files (5) 

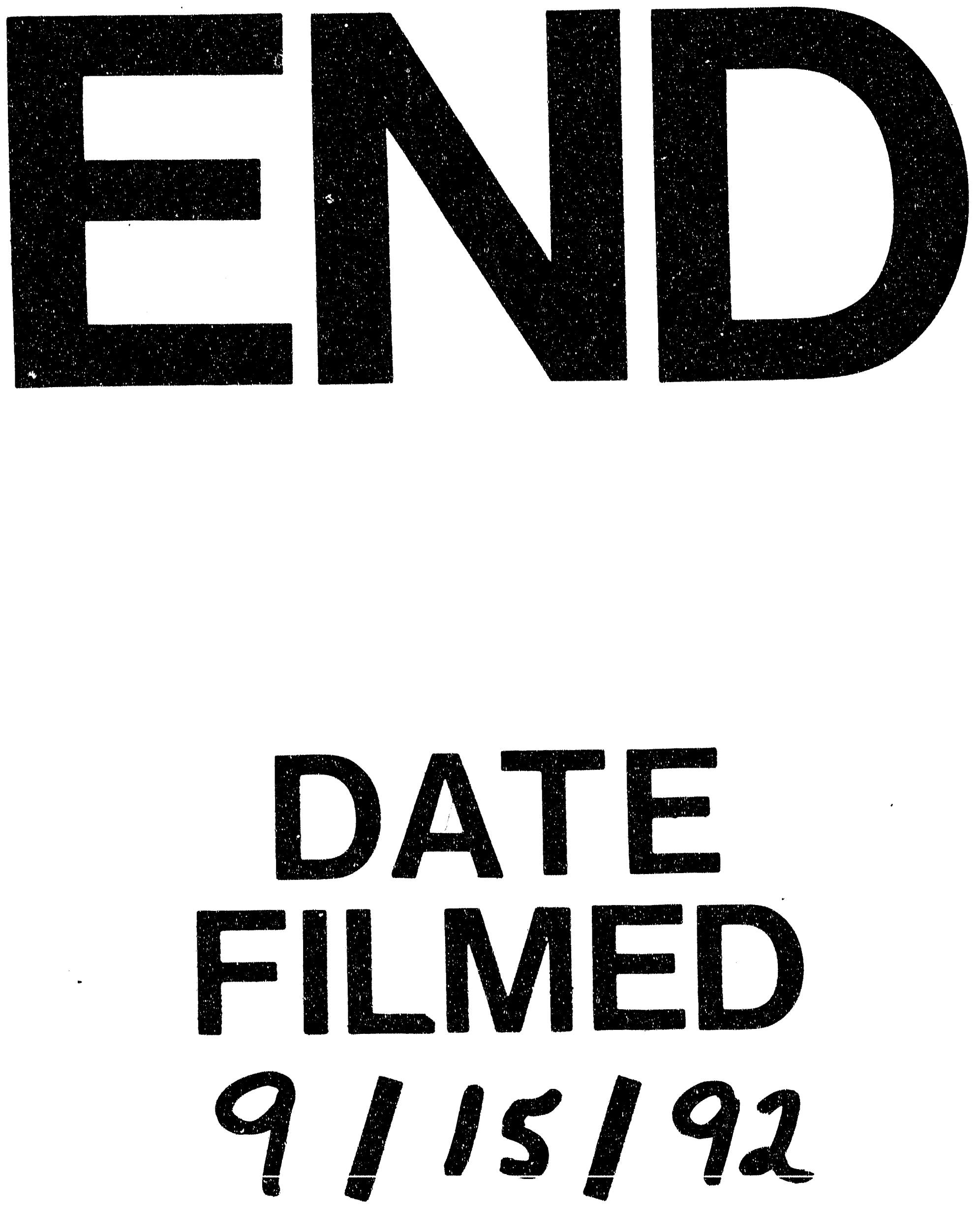
